\title{
Crystal structure and de- and rehydration behavior of two new chloride-containing Zeolitic Imidazolate Frameworks
}

-Supporting Information-

\author{
Stephan Glante ${ }^{\mathrm{a}}$, Sebastian Bette ${ }^{\mathrm{b} *}$, Gianpiero Gallo ${ }^{\mathrm{b}, \mathrm{c}}$, Robert, E. Dinnebier ${ }^{\mathrm{b}}$, Martin \\ Hartmann $^{\mathrm{a}^{*}}$
}

${ }^{a}$ Erlangen Catalysis Resource Center, Friedrich-Alexander-Universität Erlangen-Nürnberg, Egerlandstraße 3, 91058 Erlangen, Germany

${ }^{b}$ Max Planck Institute for Solid State Research, Heisenbergstraße 1, 70569 Stuttgart, Germany

'Department of Chemistry and Biology “A. Zambelli”, University of Salerno, Via Giovanni Paolo II, 132, Fisciano (SA) 84084, Italy.

\section{Experimental details and analysis methods:}

Thermal analysis. Thermal analysis was carried out using a STA 449 F5-Jupiter (Netzsch) device for TG and differential thermo analytic (DTA) measurements. $29.8 \mathrm{mg}$ of the hydrate sample were placed in $\mathrm{Al}_{2} \mathrm{O}_{3}$ crucible and heated up from $30{ }^{\circ} \mathrm{C}$ to $600{ }^{\circ} \mathrm{C}$ with a heating rate of $2{ }^{\circ} \mathrm{C} / \mathrm{min}$ in a $20 \mathrm{~mL} / \mathrm{min} \mathrm{O}_{2}$-stream. An empty crucible was used as reference material. After cooling down to room temperature the residue of the thermal decomposition was transferred into a $0.5 \mathrm{~mm}$ diameter borosilicate glass capillary (Hilgenberg glass No. 14), which was sealed and analyzed by XRPD (see below).

ATR-FTIR. Infrared spectra were measured with a JASCO 4100 IR spectrometer equipped with a PIKE GladiATR unit in a range between 400 and $4000 \mathrm{~cm}^{-1}$ with a resolution of $2 \mathrm{~cm}^{-1}$. Each spectrum is an accumulation of 16 scans.

SEM. Scanning electron microscopy measurements were carried out with a Carl-Zeiss Gemini Ultra 55, were an acceleration voltage of $1 \mathrm{kV}$ and a working distance of $5 \mathrm{~cm}$ was used.

Preliminary X-ray powder diffraction. For comparison with other known ZIF-8 (pseudo) polymorphs, XRPD patterns were collected with a Panalytical Empyrean diffractometer $\left(\lambda_{\mathrm{CuK} \alpha}=\right.$ $1.5406 \AA, \beta-N i$ filter) with a spinner setup in a range of $2 \theta=2^{\circ}$ to $50^{\circ}$ with a step size of $0.02^{\circ}$ and an exposure time of 5 seconds per step. A dehydration/rehydration experiment was carried out with the same instrument equipped with an Anton Paar $\mathrm{CHC}^{+}$chamber with z-axis scan in a range of $2 \theta=14^{\circ}$ to $15.8^{\circ}$ with a step size of $0.0066^{\circ}$ and an illumination time of 10.2 seconds 
per step. The humidity of the gas flow was adjusted with the help of a modulated humidity Generator from Pro Umid. The dehydration was carried out at $90{ }^{\circ} \mathrm{C}$ with a relative humidity of $0 \%$ and the rehydration was carried out at $30{ }^{\circ} \mathrm{C}$ with $50 \%$ relative humidity.

High resolution X-ray powder diffraction. The XRPD pattern of the ZIF-yqt hydrate compound used for crystal structure solution and Rietveld refinement was collected at room temperature on a laboratory powder diffractometer in Debye-Scherrer geometry (Stadi PDiffractometer (Stoe), $\mathrm{Cu}-\mathrm{K}_{\alpha 1}$ radiation from primary $\mathrm{Ge}(111)-J o h a n n s o n-t y p e$ monochromator, an array of 3 Mythen $2 \mathrm{~K}$ detectors (Dectris)). The sample was gently ground and filled in a 0.5 mm diameter borosilicate glass capillary (Hilgenberg glass No. 14), which was spun during the measurements. A total scan time of 1 hour was applied. The measurement was carried out multiple times. For structure solution in total 8 measurements were added up, yielding a cumulated total scan time of 8 hours.

By using the same device, the residue after thermal decomposition was analyzed and the pattern was measured with a $2 \theta$ range from $0^{\circ}$ to $110^{\circ}$ applying a total scan time of 1 hour.

Temperature dependent in-situ X-ray powder diffraction. Temperature dependent in-situ PXRD measurements were carried out on two laboratory powder diffractometers in DebyeScherrer geometry (Stadi P-Diffractometer (Stoe), Cu-Kal radiation from primary Ge(111)Johannson-type monochromator, array of 3 Mythen $2 \mathrm{~K}$ detectors (Dectris) and Bruker D8Advance; $\mathrm{Cu}-\mathrm{K} \alpha 1$ radiation from primary $\mathrm{Ge}(111)-J o h a n n-t y p e$ monochromator, Våntec detector). For the experiment run on the Stoe diffractometer, the sample was loaded into a $0.5 \mathrm{~mm}$ diameter Borosilicate glass capillary (Hilgenberg glass No. 14), which was spun during the measurements. The patterns were measured in a $2 \theta$ range from $2.0^{\circ}$ to $115.0^{\circ}$ applying a total scan time of 3 hours per measurement. The temperature was adjusted using an Oxford Cryostream device (Oxford Cryostream 500, Oxford Cryosystems). The sample was heated from $30{ }^{\circ} \mathrm{C}$ to $220^{\circ} \mathrm{C}$ in steps of $10 \mathrm{~K}$. During each step a diffraction pattern was collected, after a delay time of 10 minutes to ensure thermal equilibration of the sample. The experiment was carried out twice. In the first run the capillary was sealed and in the second run the capillary was open and therefore the sample was exposed to the dry nitrogen stream.

For the experiment run on the D8 diffractometer (Bruker), the sample was loaded into a $0.5 \mathrm{~mm}$ diameter quartz capillary, which was spun during the measurements. The patterns were measured in a $2 \theta$ range from $5.0^{\circ}$ to $40.0^{\circ}$ applying a total scan Time of 2 hours per 
measurement. The temperature was adjusted using a water-cooled furnace (mri capillary heater, $\left.(25-1000){ }^{\circ} \mathrm{C}\right)$. The sample was heated from $30{ }^{\circ} \mathrm{C}$ to $550{ }^{\circ} \mathrm{C}$ in steps of $20 \mathrm{~K}$. During each step a diffraction pattern was collected, after a delay time of 10 minutes to ensure thermal equilibration of the sample.

Structures determination and Rietveld refinements. For the solution and the refinement of the crystal structures the program TOPAS $6.0^{1}$ was used. Indexing of ZIF-yqt hydrate and ZIFyqt anhydrous was carried out by an iterative use of singular value decomposition (LSI) as implemented in TOPAS $6.0^{2}$ leading to $C$-centered monoclinic unit cell with the lattice parameters reported in Table S1 in the supporting information. From the observed systematic absences of reflections $C c$ and $C 2 / c$ were determined as most probable space groups. The peak profile was determined by a Pawley refinement ${ }^{3}$ using the fundamental parameter approach as implemented in TOPAS. ${ }^{4}$ The background was modelled using Chebyshev polynomials of $6^{\text {th }}$ order. The hump in the background of the diffraction pattern of ZIF-yqt anhydrous caused by the glass capillary was modelled with a very broad Lorentzian type peak. The crystal structures were determined by applying the global optimization method of simulated annealing (SA) ${ }^{5}$ in real space. In both cases, an attempt to reduce the symmetry was made by refining the structures with the space group $C \mathrm{c}$ which, however, did not lead to a significant improvement of the fit.

The methylimidazolate ligands were constrained by using rigid bodies in z-matrix notation, which were freely rotated and translated through the unit cell. The bond lengths and angles, were taken from related crystal structures. ${ }^{6,7}$ Atoms located on identical positions were identified by using the method of fractional occupancy merging with a merging radius of $0.7 \AA .^{8}$ The simulated annealing process was carried out iteratively. After a few hours the positions of the zinc atoms were found and fixed. The positions and orientations of the ligands were found in subsequent runs. In the structure of the hydrate phase, an occupational disorder between water molecules and chloride ions was detected by inspection of the difference Fourier map. Eventually, for both structures, the background, all lattice parameters, rotations, translations, bond lengths and angles of the rigid body, the atomic positions of zinc, water and chloride as well as the site occupancy factor (S.O.F.) of the non-coordinated water molecule (for the hydrate phase) were subsequently refined without any constraints during the final Rietveld refinement. ${ }^{9}$ Hydrogen sites were omitted due to the limitations of the powder diffraction method. The final agreement factors are listed in Table S1 (Supporting Information), the atomic coordinates and 
selected bond distances are given in Table S2 and Table S3 in the Supporting Information, the fits of the whole powder patterns are shown in Figures S1 and S2 in the supporting information. The crystallographic data have been deposited at the CCDC, deposit numbers: 18788051878806.

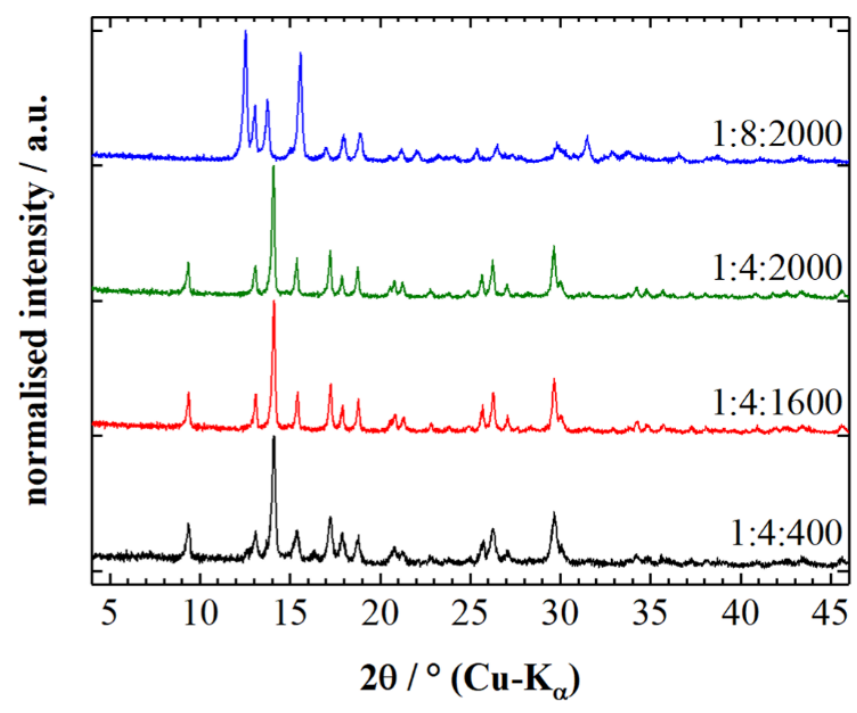

Figure S 1 Powder patterns of ZIF-yqt hydrate and ZIF-8dia, synthesized with different concentrations and ratios. The ratio between zinc chloride and 2-methylimidazole is more important than the overall concentration of the two ingredients.

Table S 1: Cell parameters at ambient conditions of known ZIF structures with 2-methylimidazole linker in comparison with ZIF-yqt hydrate.

\begin{tabular}{ccccc}
\hline & ZIF- $^{6}$ & ZIF-L $^{10}$ & ZIF-8dia $^{11}$ & ZIF-yqt hydrate $^{10}$ \\
\hline Space group & $I-43 m(217)$ & $\underline{C m c a}(64)$ & $P 2 / c(14)$ & $C 2 / c(15)$ \\
$a / \AA$ & 16.991 & 24.119 & 17.555 & 13.157 \\
$b / \AA$ & 16.991 & 17.060 & 7.731 & 16.496 \\
$c / \AA$ & 16.991 & 19.740 & 14.804 & 13.640 \\
$\alpha /{ }^{\circ}$ & 90 & 90 & 90 & 90 \\
$\beta /{ }^{\circ}$ & 90 & 90 & 107.97 & 119.17 \\
$\gamma /{ }^{\circ}$ & 90 & 90 & 90 & 90 \\
$V / \AA^{3}$ & 4905.2 & 8122.6 & 1916.3 & 2582.2 \\
\hline
\end{tabular}

The XRPD measurements (Figure S 2) show that the pattern of ZIF-yqt hydrate differs distinctively from that of known ZIF structures with 2-methylimidazole linker. We were able to 
index the pattern with a monoclinic unit cell $(C 2 / c)$ and cell parameters, which significantly differ from all other known ZIF-structures. A comparison of cell parameters is collected in Table S 1. ZIF-8 crystallizes in a cubic lattice with sodalite cages as structural motifs. The structure of ZIF-L can be understood as a combination of slicing, shifting and stacking of these cages. The zinc atoms of ZIF-8dia form a distorted diamond like net, where the methylimidazolate linkers are the main reason for this distortion. Due to the differences in the crystal lattices, ZIF-yqt hydrate (Figure S 2, d) can be clearly distinguished from other known ZIF-8-related compounds (Figure S 2, a-c) by XRPD analysis.

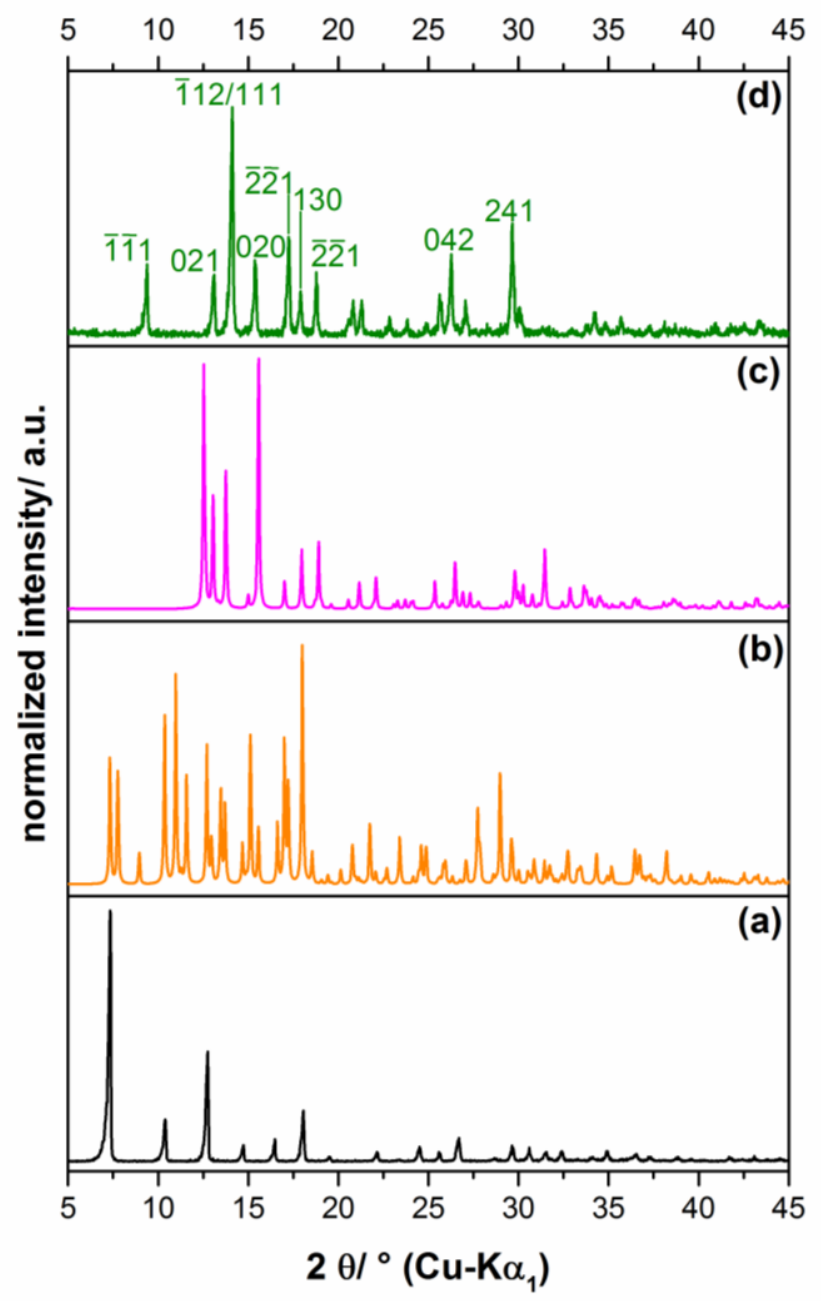

Figure $\mathbf{S} 2$ Comparison of experimental XRPD patterns of different ZIF-compounds: (a) ZIF-8, (b) ZIF-L, (c) ZIF8dia, (d) ZIF-yqt hydrate. Selected reflections are indexed according to the space group $C 2 / c$. 


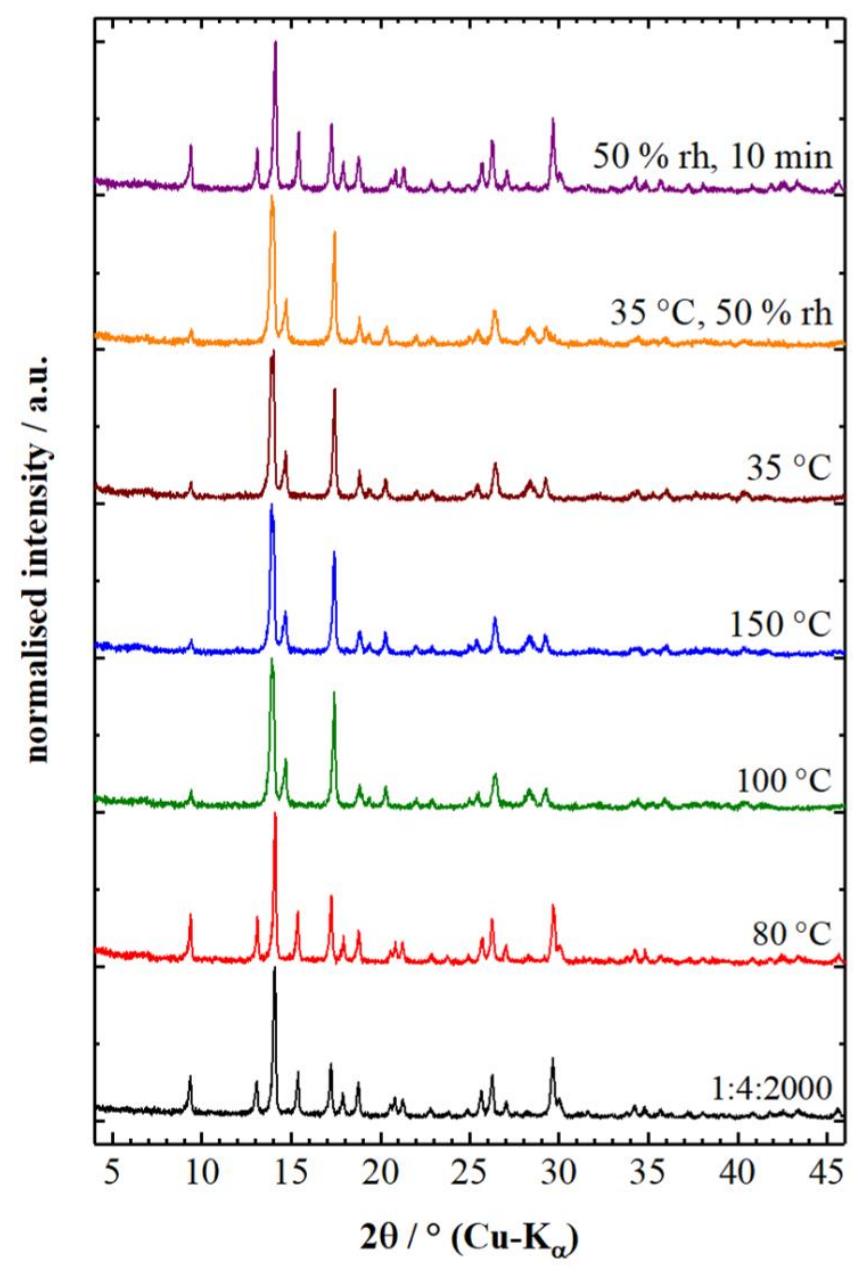

Figure S 3 Powder patterns of ZIF-yqt hydrate at different temperatures. The as-synthesized sample (black) was heated to $80{ }^{\circ} \mathrm{C}$ (red) in dry nitrogen flow in the $\mathrm{CHC}^{+}$-Chamber. At $100{ }^{\circ} \mathrm{C}$ the structural change is observed (green). Heating to $150{ }^{\circ} \mathrm{C}$ shows no difference (blue), just as cooling down to $35^{\circ} \mathrm{C}$ in dry nitrogen flow (brown). The change to a gas flow with $50 \%$ relative humidity shows no change initially (orange). After 10 min in humid gas flow, the powder pattern changes back (purple) to the shape of the pattern of the as-synthesized sample 


\section{Additional tables and figures}

Table S 2. Crystallographic and Rietveld Refinement data of the ZIF-yqt hydrate and ZIF-yqt anhydrous compound

\begin{tabular}{|c|c|c|}
\hline Compound & ZIF-yqt hydrate & ZIF-yqt anhydrous \\
\hline Molecular Formula & $\begin{array}{c}{\left[\mathrm{Zn}\left(\mathrm{C}_{4} \mathrm{~N}_{2} \mathrm{H}_{5}\right)_{2}\right] \cdot} \\
2\left[\mathrm{Zn}\left(\mathrm{C}_{4} \mathrm{~N}_{2} \mathrm{H}_{5}\right)_{1.5}\left(\mathrm{Cl}_{0.5} \mathrm{H}_{2} \mathrm{O}_{0.5}\right)\right] \cdot \times \mathrm{H}_{2} \mathrm{O} \\
\text { with } \mathrm{x}=0.74\end{array}$ & $\begin{array}{c}{\left[\mathrm{Zn}\left(\mathrm{C}_{4} \mathrm{~N}_{2} \mathrm{H}_{5}\right)_{2}\right]} \\
2\left[\mathrm{Zn}\left(\mathrm{C}_{4} \mathrm{~N}_{2} \mathrm{H}_{5}\right)_{1.5} \mathrm{Cl}\right]\end{array}$ \\
\hline $\begin{array}{c}\text { Sum formula } \\
\text { Molecular weight/g mol-1 }\end{array}$ & $\begin{array}{c}\mathrm{C}_{20} \mathrm{H}_{28.48} \mathrm{Cl} \mathrm{N}_{10} \mathrm{O}_{1.74} \mathrm{Zn}_{3} \\
668.68\end{array}$ & $\begin{array}{c}\mathrm{C}_{20} \mathrm{H}_{25} \mathrm{Cl} \mathrm{N}_{10} \mathrm{Zn}_{3} \\
637.14\end{array}$ \\
\hline Crystal system & Monoclinic & Monoclinic \\
\hline Space group & $C 2 / c(15)$ & $C 2 / c(15)$ \\
\hline Wavelength/ A & 1.5406 & 1.5406 \\
\hline$a / \AA$ & $13.1574(3)$ & $14.0819(3)$ \\
\hline$b / \AA$ & $16.4959(3)$ & $14.8689(3)$ \\
\hline$c / \AA$ & $13.6403(3)$ & $14.2313(3)$ \\
\hline$\beta /^{\circ}$ & $119.166(2)$ & $120.626(1)$ \\
\hline $\mathrm{V} / \mathrm{A}^{3}$ & $2585.2(1)$ & $2564.2(1)$ \\
\hline $\mathbf{T} / \mathbf{K}$ & 298 & 518 \\
\hline $\mathbf{Z}$ & 4 & 4 \\
\hline refined parameters & 57 & 61 \\
\hline$D_{\text {calc }} / \mathrm{g} \mathrm{cm}^{-3}$ & 1.72 & 1.65 \\
\hline $\mathbf{R}_{w p} / \%$ & 3.22 & 3.26 \\
\hline $\mathbf{R}_{p} / \%$ & 2.64 & 2.78 \\
\hline $\mathbf{R}_{\text {Bragg }} / \%$ [a] & 1.57 & 1.59 \\
\hline Starting angle measured $/{ }^{\circ} 2 \vartheta$ & 0 & 0 \\
\hline Final angle measured $/{ }^{\circ} 29$ & 110 & 110 \\
\hline Starting angle used $/{ }^{\circ} 29$ & 6 & 7 \\
\hline Final angle used $/{ }^{\circ} 2 \vartheta$ & 80 & 84 \\
\hline Step width $\left({ }^{\circ} 29\right)$ & 0.01 & 0.01 \\
\hline Time(hrs) & 8 & 3 \\
\hline
\end{tabular}

${ }^{[a]}$ as defined in TOPAS 6.0 
Table S 3 Atomic coordinates of the ZIF-yqt hydrate and ZIF-yqt anhydrous compound.

\begin{tabular}{|c|c|c|c|c|c|c|c|}
\hline Atom & Wyck. & Site & S.O.F. & $x / a$ & $y / b$ & $z / c$ & $\mathbf{B} / \AA^{2}$ \\
\hline \multicolumn{8}{|c|}{ ZIF-yqt hydrate at $298 \mathrm{~K}$} \\
\hline $\mathrm{Zn} 1$ & $4 e$ & 2 & 1 & 0 & $0.9299(3)$ & $1 / 4$ & $2.62(6)$ \\
\hline $\mathrm{Zn} 2$ & $8 f$ & 1 & 1 & $0.4029(2)$ & $0.83603(17)$ & $0.9989(2)$ & $2.62(6)$ \\
\hline $\mathrm{Cl1}$ & & & 0.5 & $0.0402(4)$ & $0.5772(4)$ & $0.4794(5)$ & \\
\hline $\mathrm{O} 1$ & $8 f$ & 1 & 0.5 & $0.0402(4)$ & $0.5772(4)$ & $0.4794(5)$ & $0.15(10)$ \\
\hline $\mathrm{O} 2$ & $4 e$ & 2 & $0.74(1)$ & 0 & $0.6448(11)$ & $1 / 4$ & $4.68(23)$ \\
\hline $\mathrm{C} 11$ & $8 f$ & 1 & 1 & $0.9729(7)$ & $0.8046(6)$ & $0.3920(7)$ & $3.00(20)$ \\
\hline $\mathrm{C} 12$ & $8 f$ & 1 & 1 & $0.0974(11)$ & $0.7998(13)$ & $0.4819(11)$ & $3.00(20)$ \\
\hline $\mathrm{C} 13$ & $8 f$ & 1 & 1 & $0.8085(13)$ & $0.8363(11)$ & $0.2414(13)$ & $3.00(20)$ \\
\hline $\mathrm{C} 14$ & $8 f$ & 1 & 1 & $0.785(2)$ & $0.7797(12)$ & $0.297(2)$ & $3.00(20)$ \\
\hline N11 & $8 f$ & 1 & 1 & $0.9274(11)$ & $0.8533(5)$ & $0.2999(9)$ & $3.00(20)$ \\
\hline N12 & $8 f$ & 1 & 1 & $0.8820(11)$ & $0.7612(5)$ & $0.3858(10)$ & $3.00(20)$ \\
\hline $\mathrm{C} 21$ & $8 f$ & 1 & 1 & $0.8002(6)$ & $0.9479(4)$ & $0.6403(8)$ & $3.00(20)$ \\
\hline $\mathrm{C} 22$ & $8 f$ & 1 & 1 & $0.7791(11)$ & $0.9436(8)$ & $0.7383(12)$ & $3.00(20)$ \\
\hline $\mathrm{C} 23$ & $8 f$ & 1 & 1 & $0.7945(14)$ & $0.9291(10)$ & $0.4780(13)$ & $3.00(20)$ \\
\hline $\mathrm{C} 24$ & $8 f$ & 1 & 1 & $0.8749(14)$ & $0.9855(10)$ & $0.534(3)$ & $3.00(20)$ \\
\hline $\mathrm{N} 21$ & $8 f$ & 1 & 1 & $0.7457(7)$ & $0.9042(5)$ & $0.5436(10)$ & $3.00(20)$ \\
\hline $\mathrm{N} 22$ & $8 f$ & 1 & 1 & $0.8779(7)$ & $0.9963(5)$ & $0.6295(11)$ & $3.00(20)$ \\
\hline $\mathrm{C} 31$ & $4 e$ & 2 & 1 & 0 & $0.3481(6)$ & $1 / 4$ & $3.00(20)$ \\
\hline $\mathrm{C} 32$ & $4 e$ & 2 & 1 & 0 & $0.4387(8)$ & $1 / 4$ & $3.00(20)$ \\
\hline $\mathrm{C} 33$ & $8 f$ & 1 & 1 & $0.0202(9)$ & $0.2195(9)$ & $0.2990(17)$ & $3.00(20)$ \\
\hline N31 & $8 f$ & 1 & 1 & $0.0375(9)$ & $0.2984(8)$ & $0.3407(6)$ & $3.00(20)$ \\
\hline \multicolumn{8}{|c|}{ ZIF-yqt anhydrous at $518 \mathrm{~K}$} \\
\hline $\mathrm{Zn} 1$ & $8 f$ & 1 & 1 & $0.39447(18)$ & $0.62342(17)$ & $0.50735(18)$ & $2.71(8)$ \\
\hline $\mathrm{Zn} 2$ & $4 e$ & 2 & 1 & 0 & $0.3891(2)$ & $1 / 4$ & $2.71(8)$ \\
\hline $\mathrm{Cl} 1$ & $4 a$ & -1 & 1 & 0 & 0 & 0 & $3.00(21)$ \\
\hline $\mathrm{C} 11$ & $8 f$ & 1 & 1 & $0.8094(6)$ & $0.5271(5)$ & $0.1451(7)$ & $2.67(14)$ \\
\hline $\mathrm{C} 12$ & $8 f$ & 1 & 1 & $0.7826(11)$ & $0.5428(9)$ & $0.2328(12)$ & $2.67(14)$ \\
\hline $\mathrm{C} 13$ & $8 f$ & 1 & 1 & $0.8808(17)$ & $0.4781(14)$ & $0.0485(16)$ & $2.67(14)$ \\
\hline $\mathrm{C} 14$ & $8 f$ & 1 & 1 & $0.802(2)$ & $0.5408(17)$ & $-0.0135(19)$ & $2.67(14)$ \\
\hline N11 & $8 f$ & 1 & 1 & $0.8859(7)$ & $0.4692(6)$ & $0.1485(12)$ & $2.67(14)$ \\
\hline N12 & $8 f$ & 1 & 1 & $0.7623(8)$ & $0.5677(7)$ & $0.0458(10)$ & $2.67(14)$ \\
\hline $\mathrm{C} 21$ & $8 f$ & 1 & 1 & $0.0239(9)$ & $0.2586(8)$ & $0.1047(8)$ & $2.67(14)$ \\
\hline $\mathrm{C} 22$ & $8 f$ & 1 & 1 & $-0.0970(13)$ & $0.2414(18)$ & $0.0332(13)$ & $2.67(14)$ \\
\hline $\mathrm{C} 23$ & $8 f$ & 1 & 1 & $0.1864(15)$ & $0.3085(14)$ & $0.2320(16)$ & $2.67(14)$ \\
\hline $\mathrm{C} 24$ & $8 f$ & 1 & 1 & $0.204(3)$ & $0.2485(18)$ & $0.169(3)$ & $2.67(14)$ \\
\hline $\mathrm{N} 21$ & $8 f$ & 1 & 1 & $0.0732(14)$ & $0.3151(7)$ & $0.1919(9)$ & $2.67(14)$ \\
\hline $\mathrm{N} 22$ & $8 f$ & 1 & 1 & $0.1070(13)$ & $0.2209(7)$ & $0.0957(11)$ & $2.67(14)$ \\
\hline $\mathrm{C} 31$ & $4 e$ & 2 & 1 & 0 & $0.8956(12)$ & $1 / 4$ & $2.67(14)$ \\
\hline $\mathrm{C} 32$ & $4 e$ & 2 & 1 & 0 & $0.9962(14)$ & $1 / 4$ & $2.67(14)$ \\
\hline $\mathrm{C} 33$ & $8 f$ & 1 & 1 & $0.0188(10)$ & $0.7530(15)$ & $0.298(2)$ & $2.67(14)$ \\
\hline N31 & $8 f$ & 1 & 1 & $0.0349(9)$ & $0.8406(14)$ & $0.3379(7)$ & $2.67(14)$ \\
\hline
\end{tabular}


Table S 4 Selected atomic distances of the ZIF-yqt hydrate and ZIF-yqt anhydrous compounds.

\begin{tabular}{cc|cc}
\hline $\begin{array}{c}\text { Atoms } \\
\text { ZIF-yqt hydrate at 298 K }\end{array}$ & $\begin{array}{c}\text { Atoms } \\
\text { ZIF-yqt anhydrous 518 K }\end{array}$ \\
\hline $\mathrm{Zn}(1)-\mathrm{N}(11)$ & $1.899(9) \AA$ & $\mathrm{Zn}(1)-\mathrm{N}(12)$ & $2.11(1) \AA$ \\
$\mathrm{Zn}(1)-\mathrm{N}(22)$ & $2.048(6) \AA$ & $\mathrm{Zn}(1)-\mathrm{N}(22)$ & $2.06(2) \AA$ \\
$\mathrm{Zn}(2)-\mathrm{N}(12)$ & $2.077(6) \AA$ & $\mathrm{Zn}(1)-\mathrm{N}(31)$ & $1.97(1) \AA$ \\
$\mathrm{Zn}(2)-\mathrm{N}(21)$ & $2.149(8) \AA$ & $\mathrm{Zn}(1)-\mathrm{Cl}(1)$ & $2.40(1) \AA$ \\
$\mathrm{Zn}(2)-\mathrm{N}(31)$ & $2.028(4)$ & $\mathrm{Zn}(2)-\mathrm{N}(11)$ & $1.93(1) \AA$ \\
$\mathrm{Zn}(2)-\mathrm{Cl} / \mathrm{O}(1)$ & $2.418(7) \AA$ & $\mathrm{Zn}(2)-\mathrm{N}(21)$ & \\
$\mathrm{Cl} / \mathrm{O}(1)-\mathrm{Cl} / \mathrm{O}(1)$ & $2.916(10) \AA$ & & \\
$\mathrm{Cl} / \mathrm{O}(1)-\mathrm{O}(2)$ & $3.110(10) \AA$ & & \\
\hline
\end{tabular}

The diffraction patterns taken during the temperature depended in-situ XRPD studies in an open (from $30^{\circ} \mathrm{C}$ to $140{ }^{\circ} \mathrm{C}$ ) and sealed capillary (from $30{ }^{\circ} \mathrm{C}$ to $80^{\circ} \mathrm{C}$ ) were used for Rietveld refinements of the crystal structure of ZIF-yqt hydrate with different contents of non-coordinated hydrate water. The fits of the whole powder patterns are shown in Figure S 6 to S21. The refinements were carried out as described above. The crystallographic data are attached to the supporting information

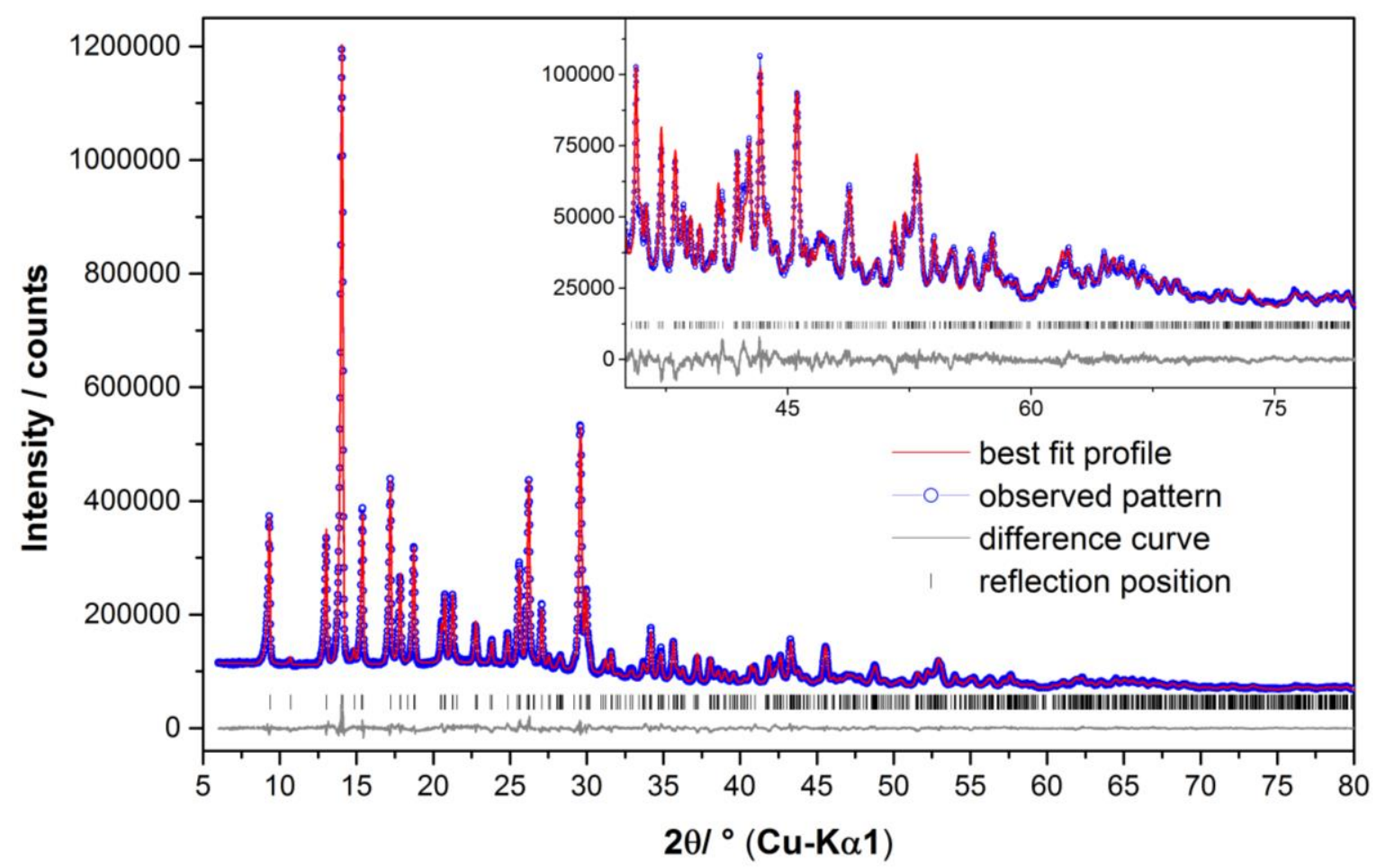

Figure S 4 Scattered X-ray intensities of ZIF-yqt hydrate at ambient conditions as a function of diffraction angle 20. The observed pattern (circles) measured in Debye-Scherrer geometry, the best Rietveld fit profiles (line) and the difference curve between the observed and the calculated profiles (below) are shown. The high angle part starting at $30.5^{\circ}$ in $2 \theta$ is enlarged for clarity. 


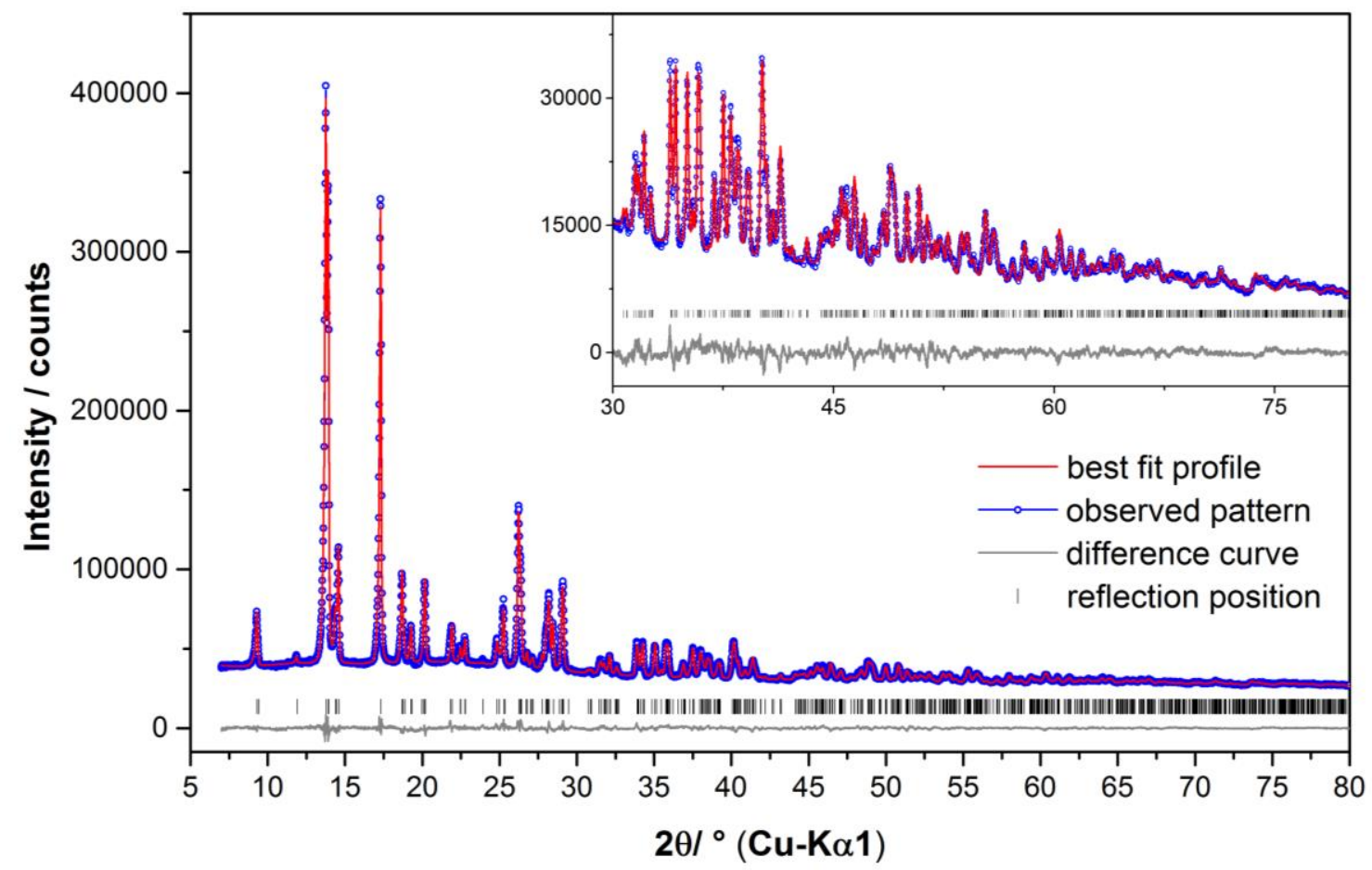

Figure S 5 Scattered X-ray intensities of ZIF-yqt anhydrous at $220{ }^{\circ} \mathrm{C}$ as a function of diffraction angle $2 \theta$. The observed pattern (circles) measured in Debye-Scherrer geometry, the best Rietveld fit profiles (line) and the difference curve between the observed and the calculated profiles (below) are shown. The high angle part starting at $30^{\circ}$ in $2 \theta$ is enlarged for clarity 


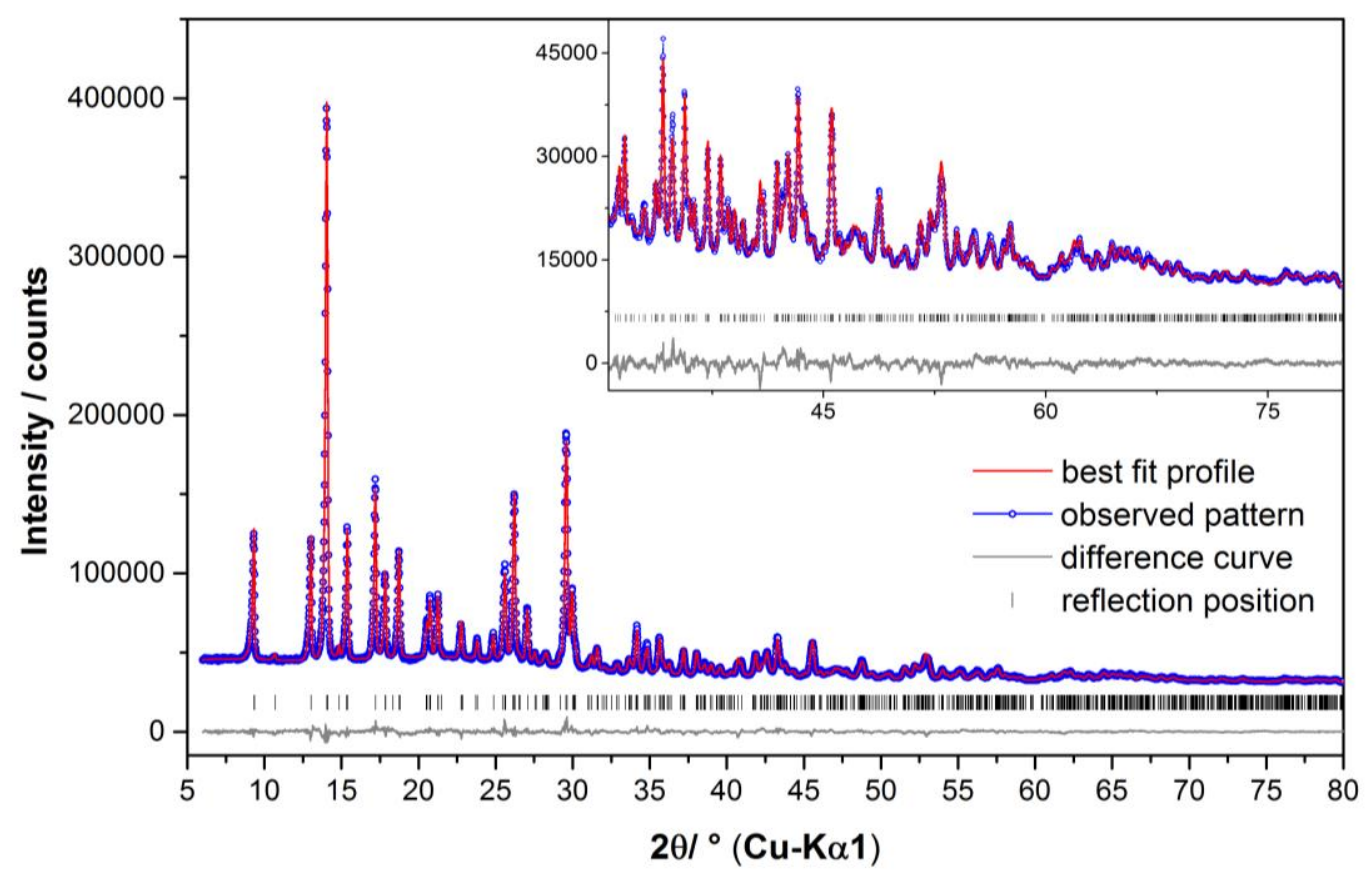

Figure S 6 Scattered X-ray intensities of ZIF-yqt hydrate at $30{ }^{\circ} \mathrm{C}$ measured in a sealed capillary as a function of diffraction angle 20. The observed pattern (circles) measured in Debye-Scherrer geometry, the best Rietveld fit profiles (line) and the difference curve between the observed and the calculated profiles (below) are shown. The high angle part starting at $30.5^{\circ}$ in $2 \theta$ is enlarged for clarity.

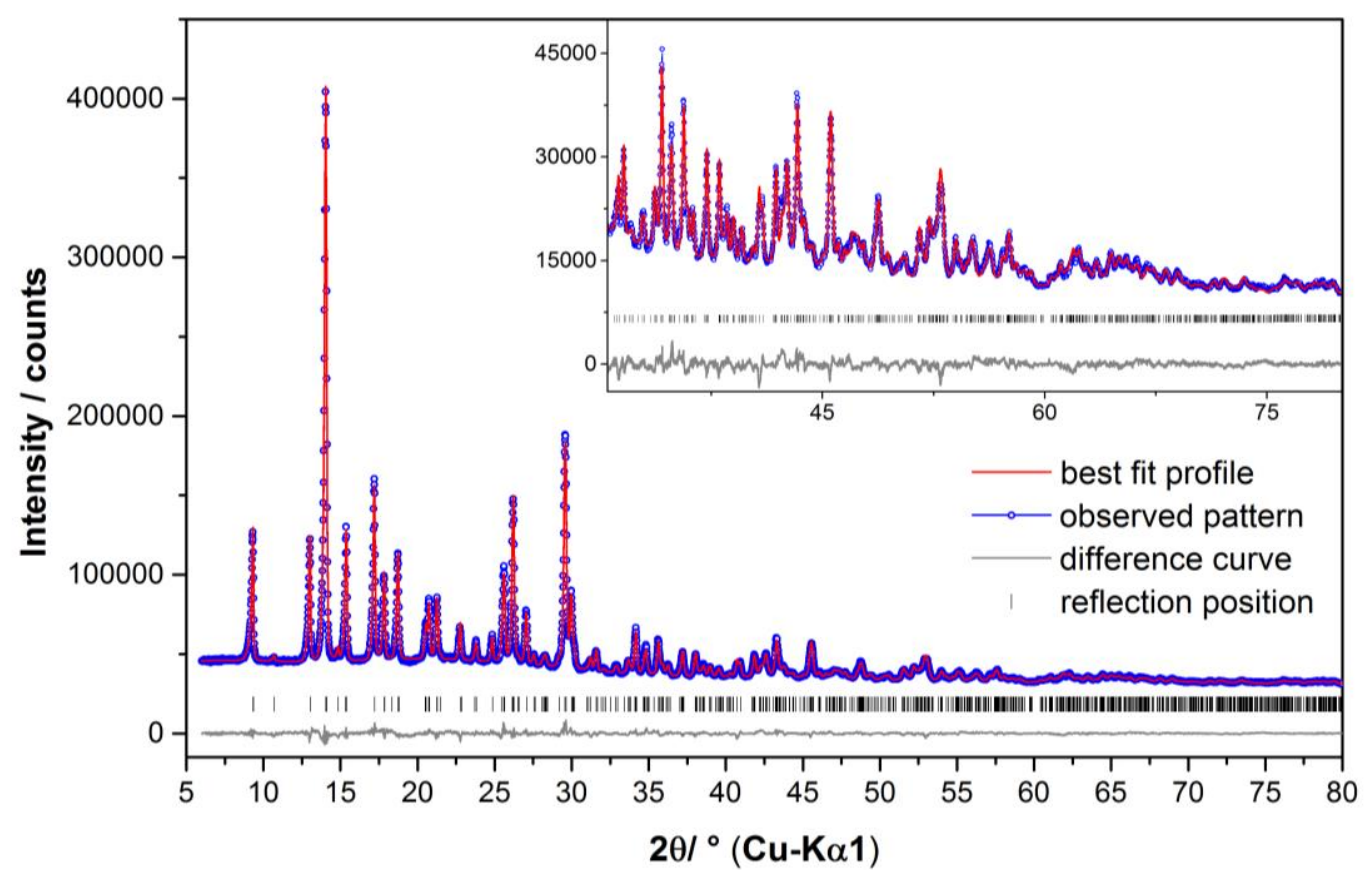

Figure $\mathbf{S} 7$ Scattered X-ray intensities of ZIF-yqt hydrate at $40{ }^{\circ} \mathrm{C}$ measured in a sealed capillary as a function of diffraction angle 2 $\theta$. The observed pattern (circles) measured in Debye-Scherrer geometry, the best Rietveld fit profiles (line) and the difference curve between the observed and the calculated profiles (below) are shown. The high angle part starting at $30.5^{\circ}$ in $2 \theta$ is enlarged for clarity. 


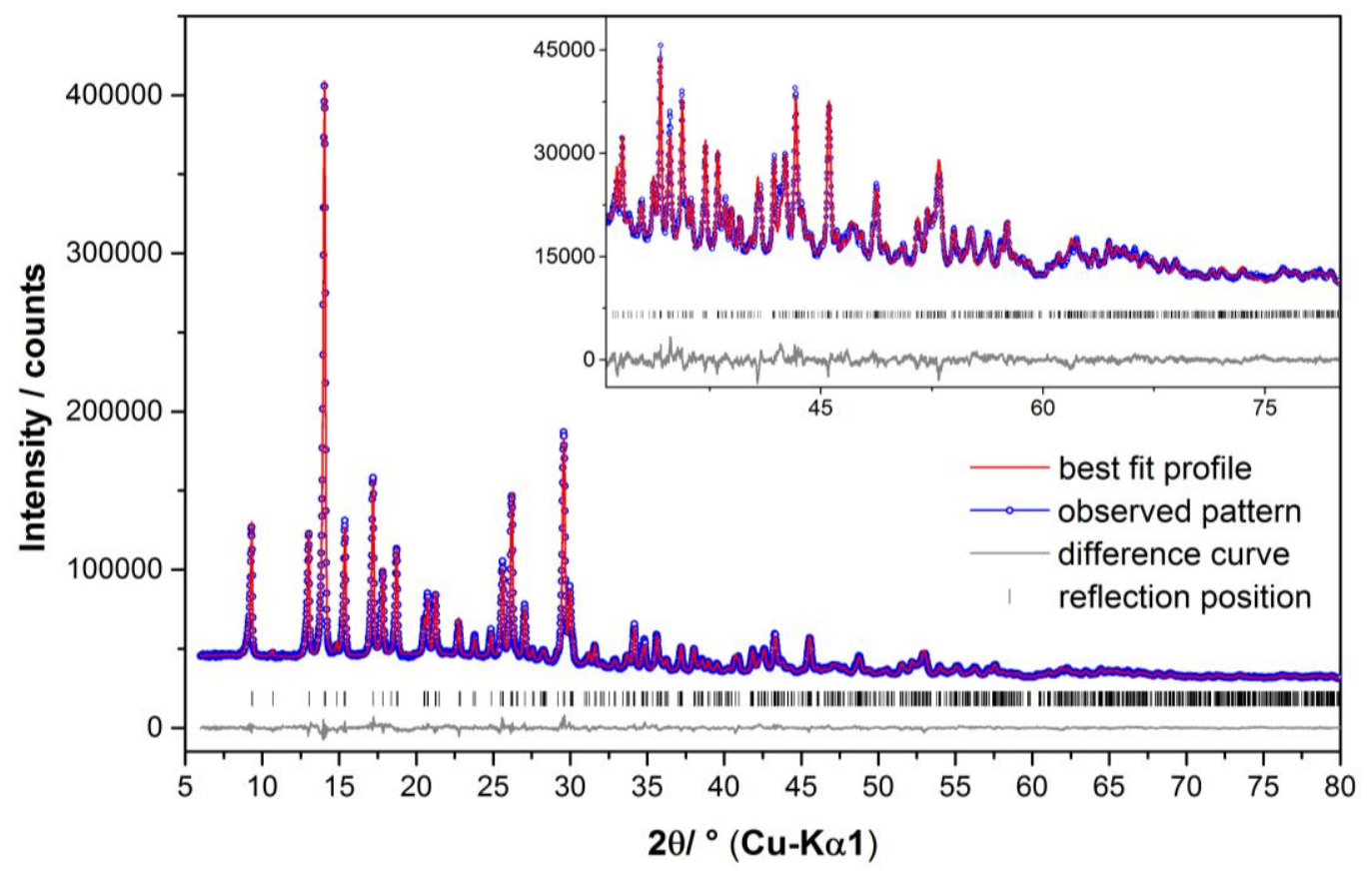

Figure S 8 Scattered X-ray intensities of ZIF-yqt hydrate at $50{ }^{\circ} \mathrm{C}$ measured in a sealed capillary as a function of diffraction angle 20. The observed pattern (circles) measured in Debye-Scherrer geometry, the best Rietveld fit profiles (line) and the difference curve between the observed and the calculated profiles (below) are shown. The high angle part starting at $30.5^{\circ}$ in $2 \theta$ is enlarged for clarity.

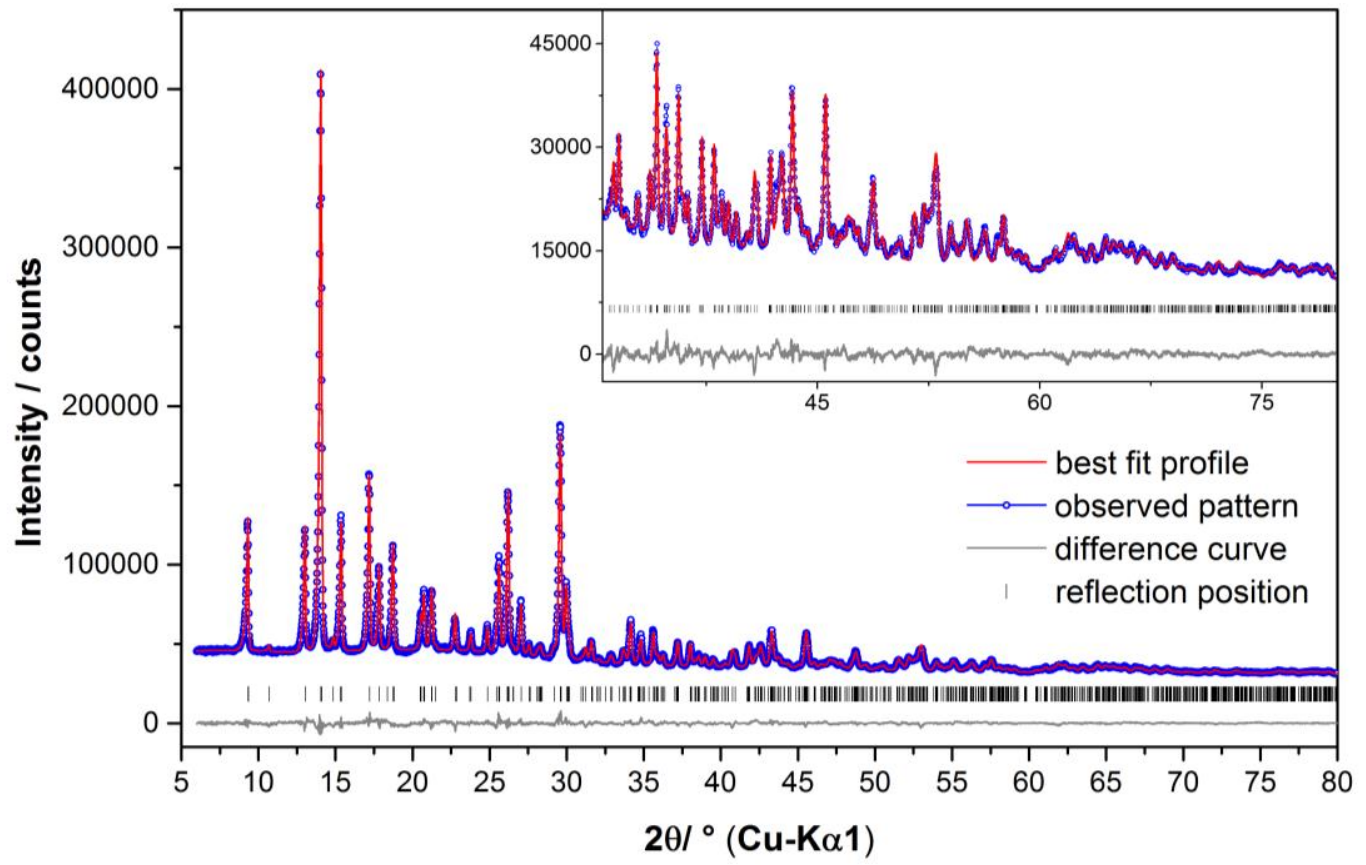

Figure S 9 Scattered X-ray intensities of ZIF-yqt hydrate at $60{ }^{\circ} \mathrm{C}$ measured in a sealed capillary as a function of diffraction angle 20. The observed pattern (circles) measured in Debye-Scherrer geometry, the best Rietveld fit profiles (line) and the difference curve between the observed and the calculated profiles (below) are shown. The high angle part starting at $30.5^{\circ}$ in $2 \theta$ is enlarged for clarity. 


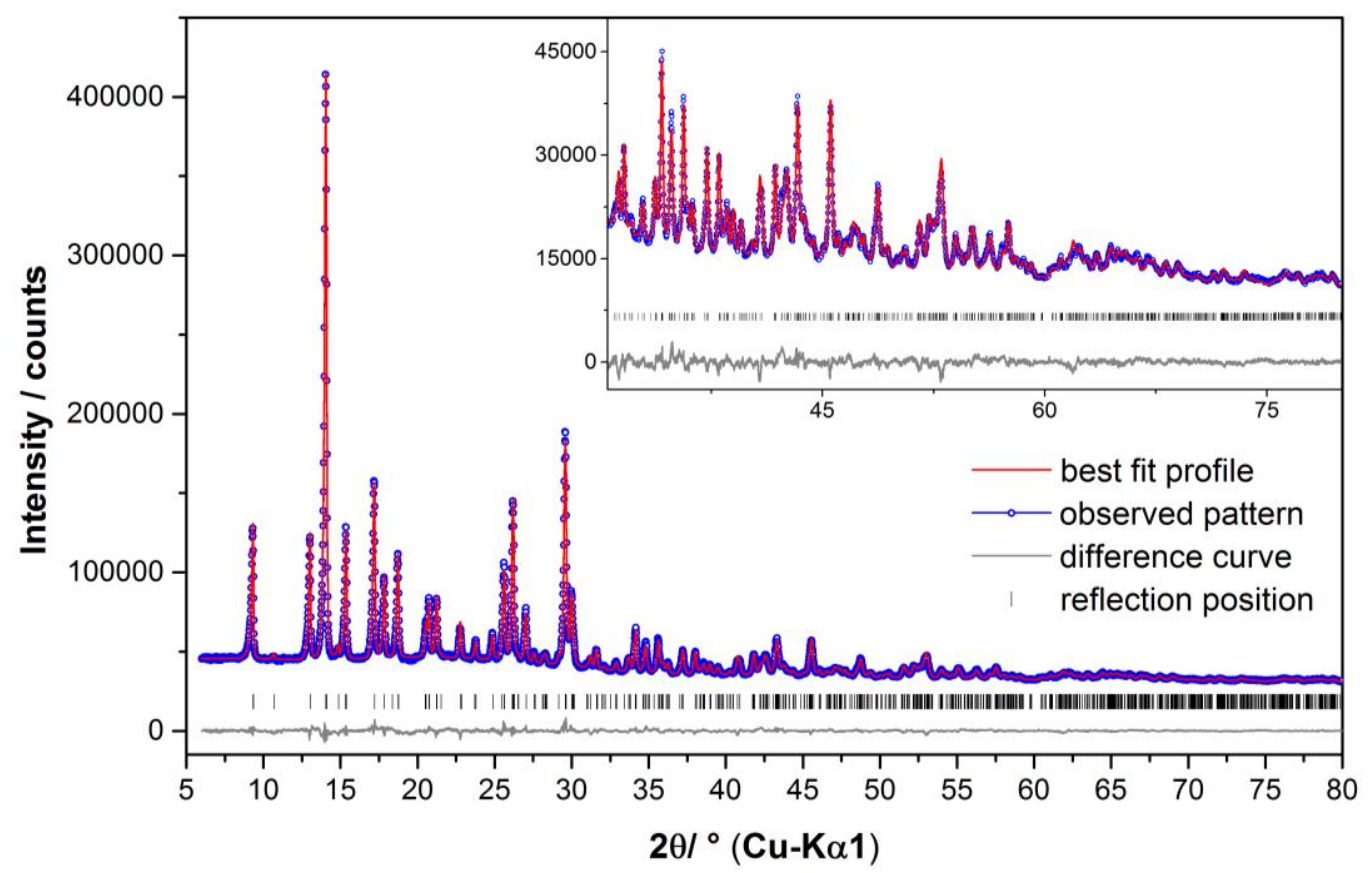

Figure S 10 Scattered X-ray intensities of ZIF-yqt hydrate at $70{ }^{\circ} \mathrm{C}$ measured in a sealed capillary as a function of diffraction angle 20. The observed pattern (circles) measured in Debye-Scherrer geometry, the best Rietveld fit profiles (line) and the difference curve between the observed and the calculated profiles (below) are shown. The high angle part starting at $30.5^{\circ}$ in $2 \theta$ is enlarged for clarity.

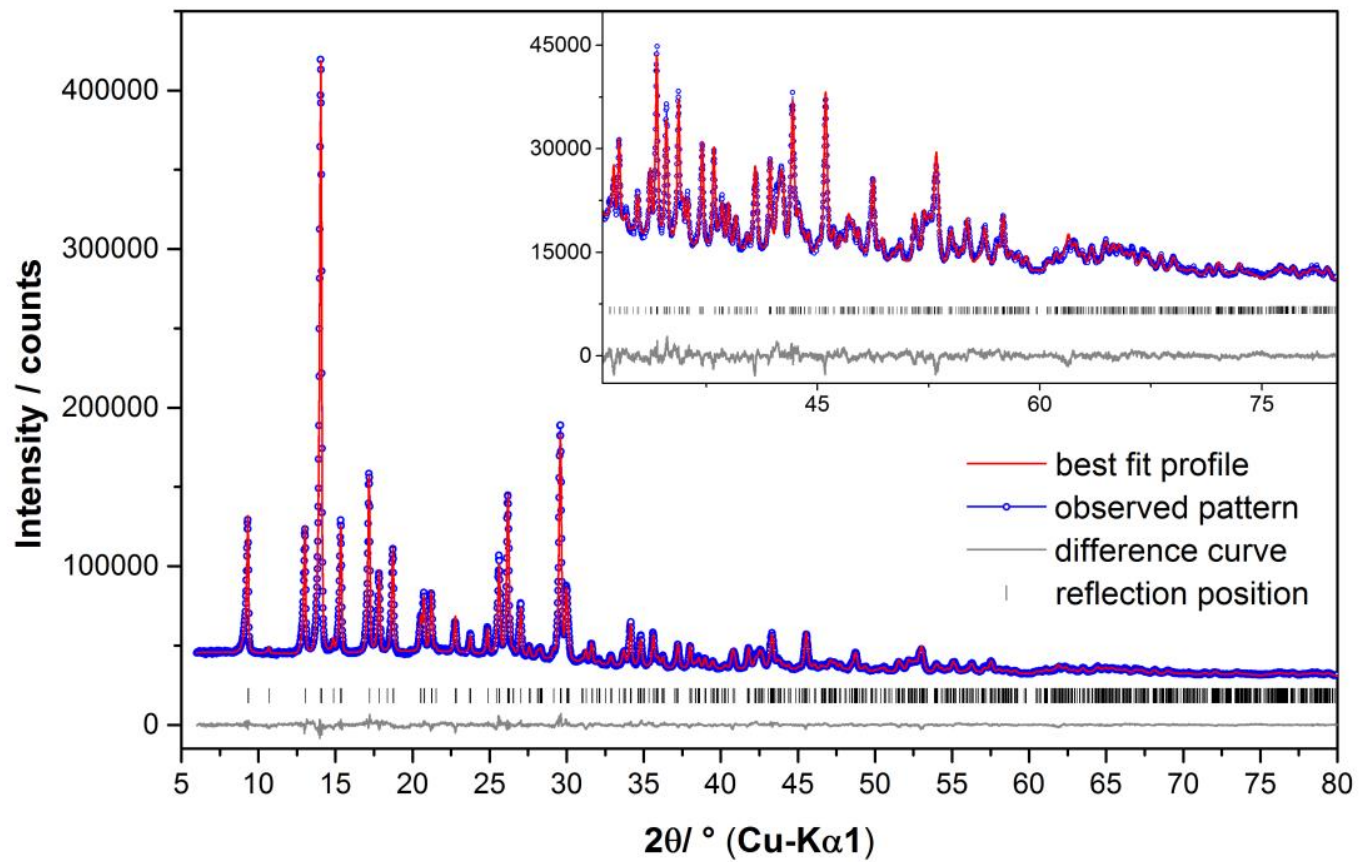

Figure S 11 Scattered X-ray intensities of ZIF-yqt hydrate at $80{ }^{\circ} \mathrm{C}$ measured in a sealed capillary as a function of diffraction angle 20. The observed pattern (circles) measured in Debye-Scherrer geometry, the best Rietveld fit profiles (line) and the difference curve between the observed and the calculated profiles (below) are shown. The high angle part starting at $30.5^{\circ}$ in $2 \theta$ is enlarged for clarity. 


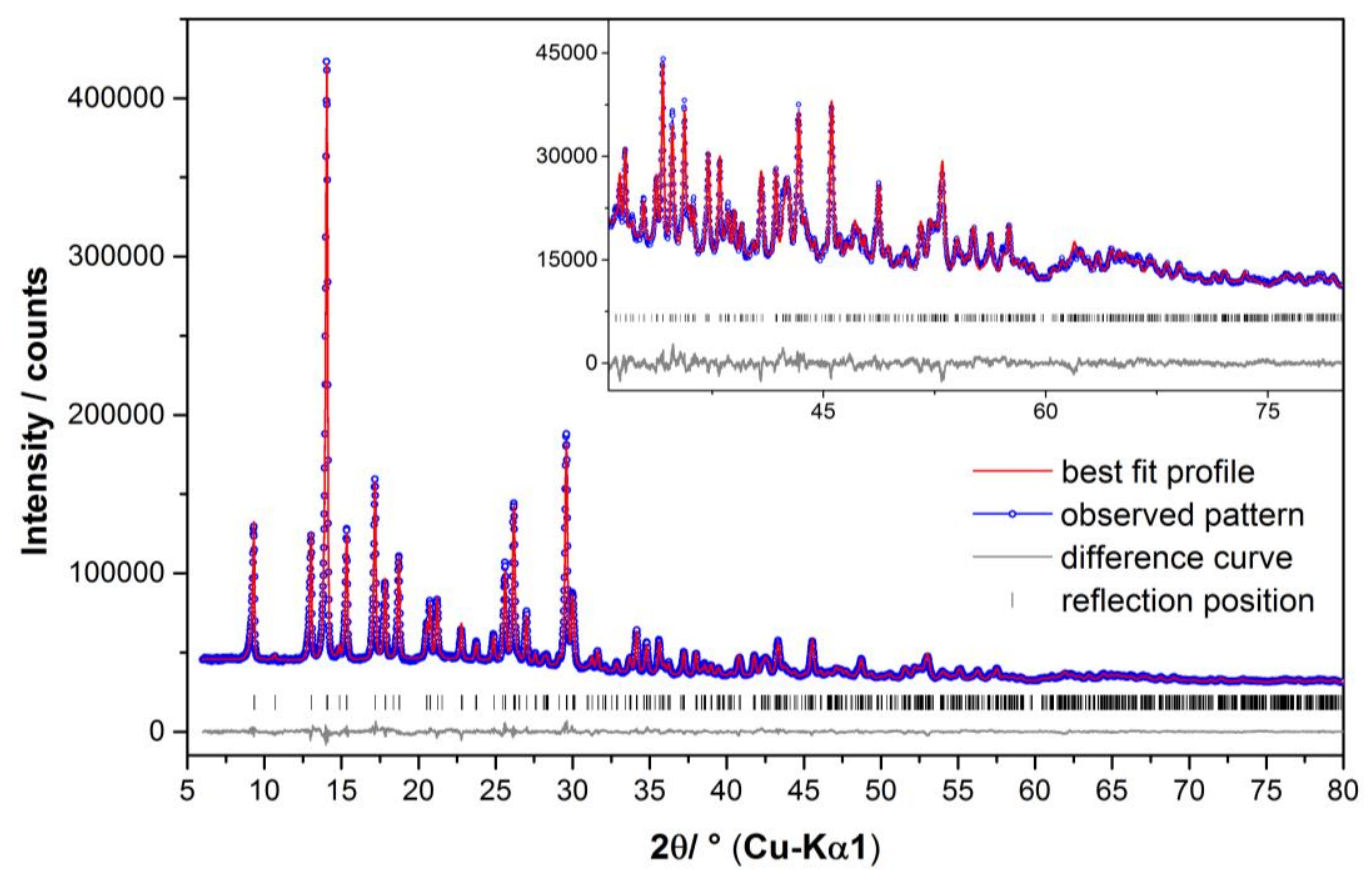

Figure S 12 Scattered X-ray intensities of ZIF-yqt hydrate at $90{ }^{\circ} \mathrm{C}$ measured in a sealed capillary as a function of diffraction angle 2 $\theta$. The observed pattern (circles) measured in Debye-Scherrer geometry, the best Rietveld fit profiles (line) and the difference curve between the observed and the calculated profiles (below) are shown. The high angle part starting at $30.5^{\circ}$ in $2 \theta$ is enlarged for clarity.

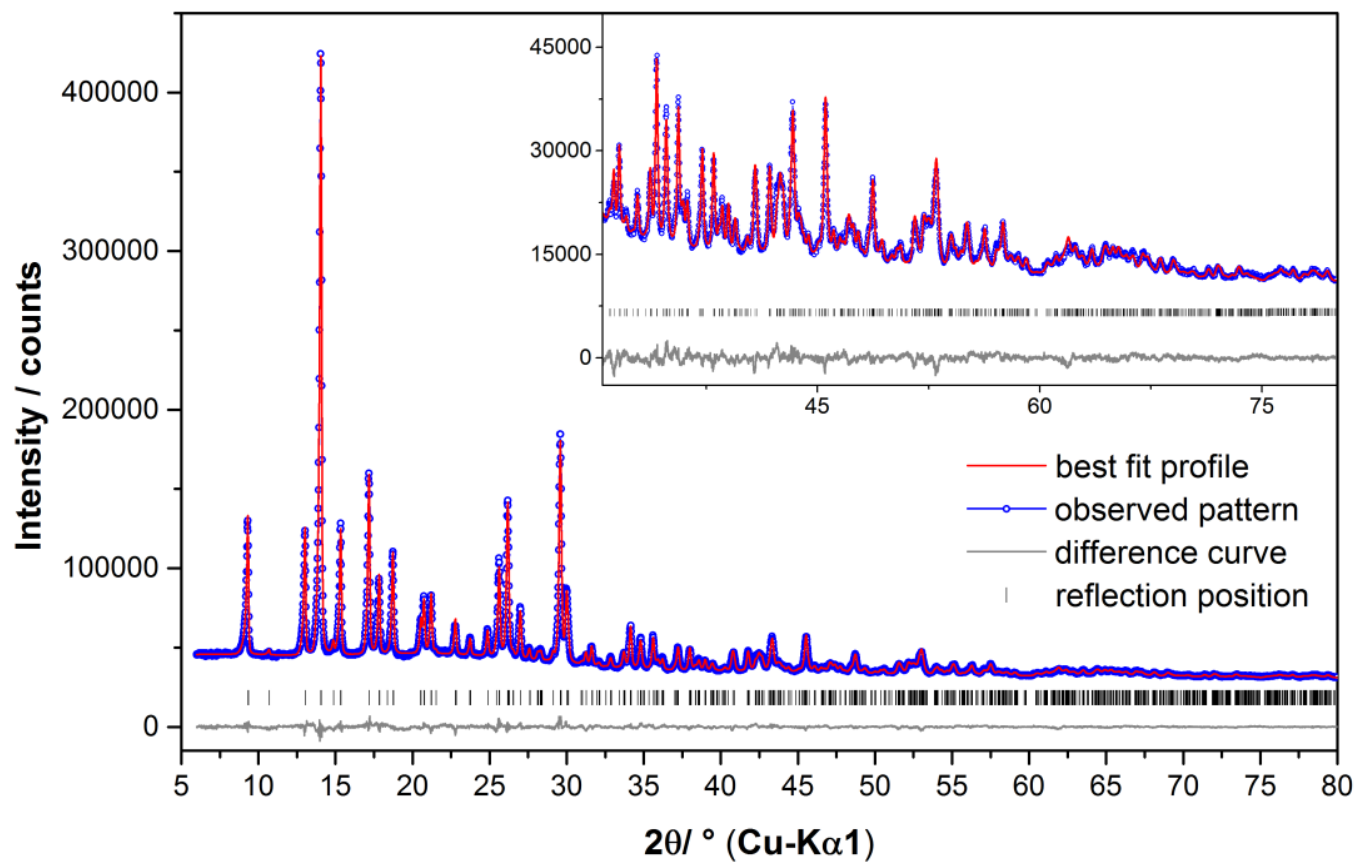

Figure S 13 Scattered X-ray intensities of ZIF-yqt hydrate at $100{ }^{\circ} \mathrm{C}$ measured in a sealed capillary as a function of diffraction angle 20. The observed pattern (circles) measured in Debye-Scherrer geometry, the best Rietveld fit profiles (line) and the difference curve between the observed and the calculated profiles (below) are shown. The high angle part starting at $30.5^{\circ}$ in $2 \theta$ is enlarged for clarity. 


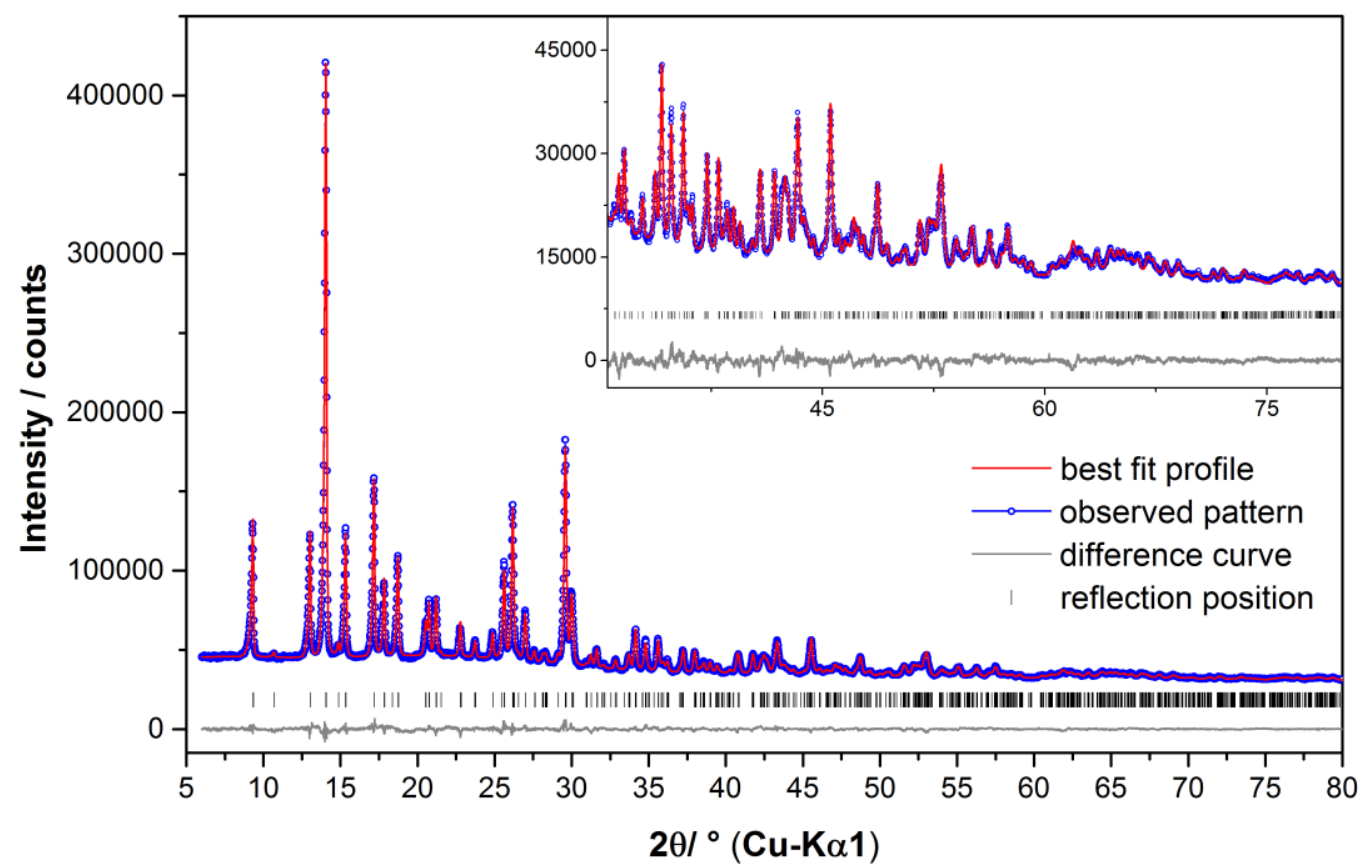

Figure S 14 Scattered X-ray intensities of ZIF-yqt hydrate at $110^{\circ} \mathrm{C}$ measured in a sealed capillary as a function of diffraction angle 2 $\theta$. The observed pattern (circles) measured in Debye-Scherrer geometry, the best Rietveld fit profiles (line) and the difference curve between the observed and the calculated profiles (below) are shown. The high angle part starting at $30.5^{\circ}$ in $2 \theta$ is enlarged for clarity.

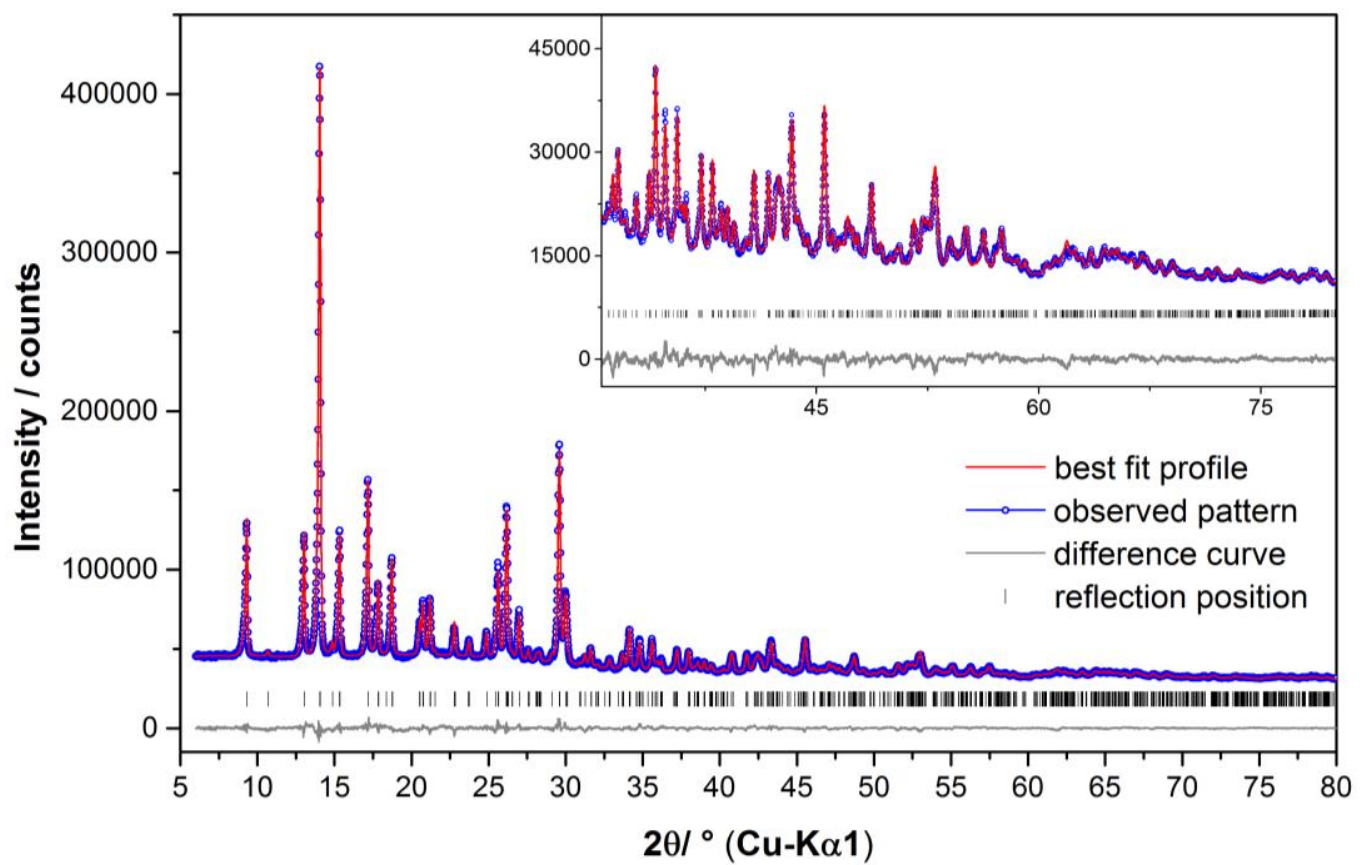

Figure S 15 Scattered X-ray intensities of ZIF-yqt hydrate at $120^{\circ} \mathrm{C}$ measured in a sealed capillary as a function of diffraction angle 20. The observed pattern (circles) measured in Debye-Scherrer geometry, the best Rietveld fit profiles (line) and the difference curve between the observed and the calculated profiles (below) are shown. The high angle part starting at $30.5^{\circ}$ in $2 \theta$ is enlarged for clarity. 


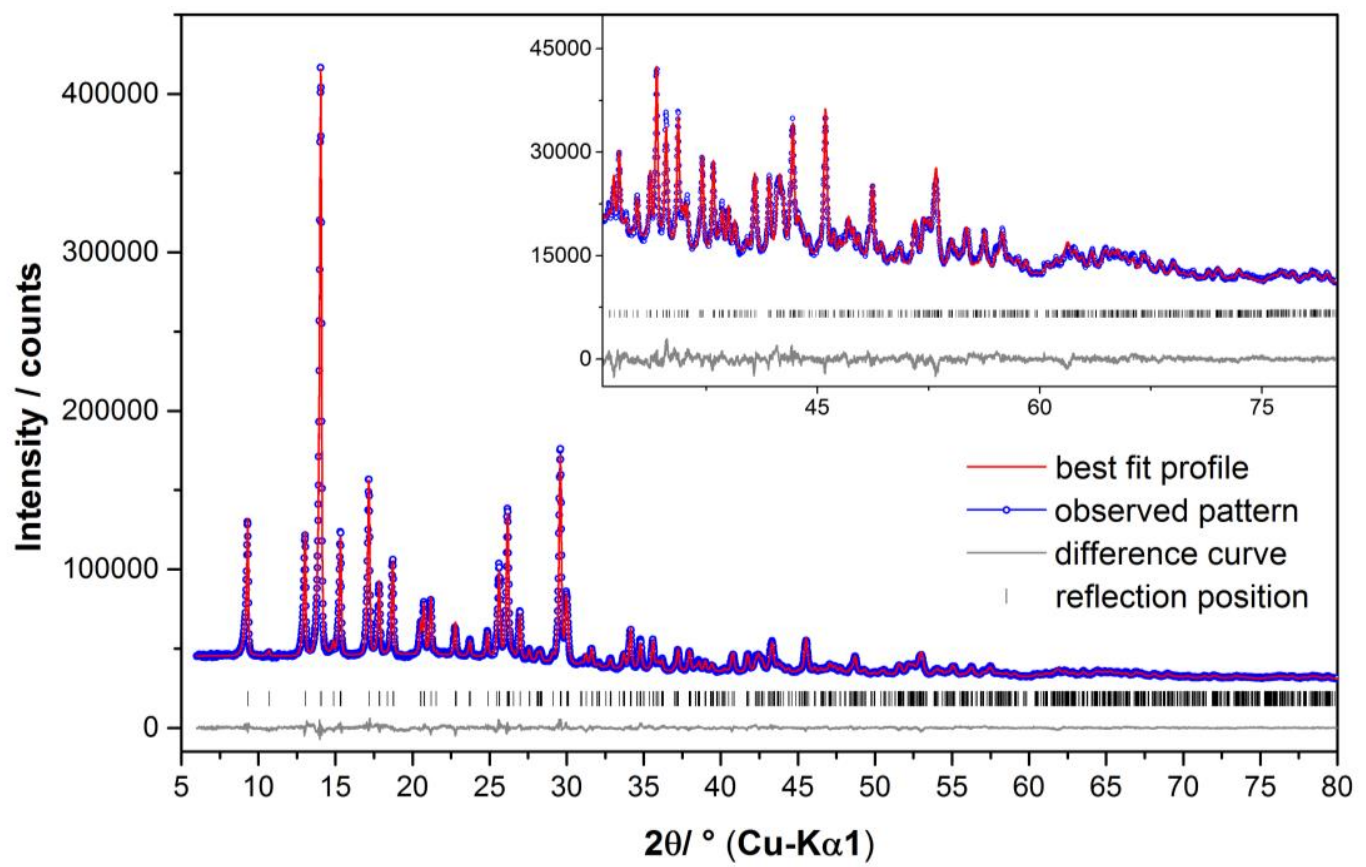

Figure S 16 Scattered X-ray intensities of ZIF-yqt hydrate at $130{ }^{\circ} \mathrm{C}$ measured in a sealed capillary as a function of diffraction angle 20. The observed pattern (circles) measured in Debye-Scherrer geometry, the best Rietveld fit profiles (line) and the difference curve between the observed and the calculated profiles (below) are shown. The high angle part starting at $30.5^{\circ}$ in $2 \theta$ is enlarged for clarity.

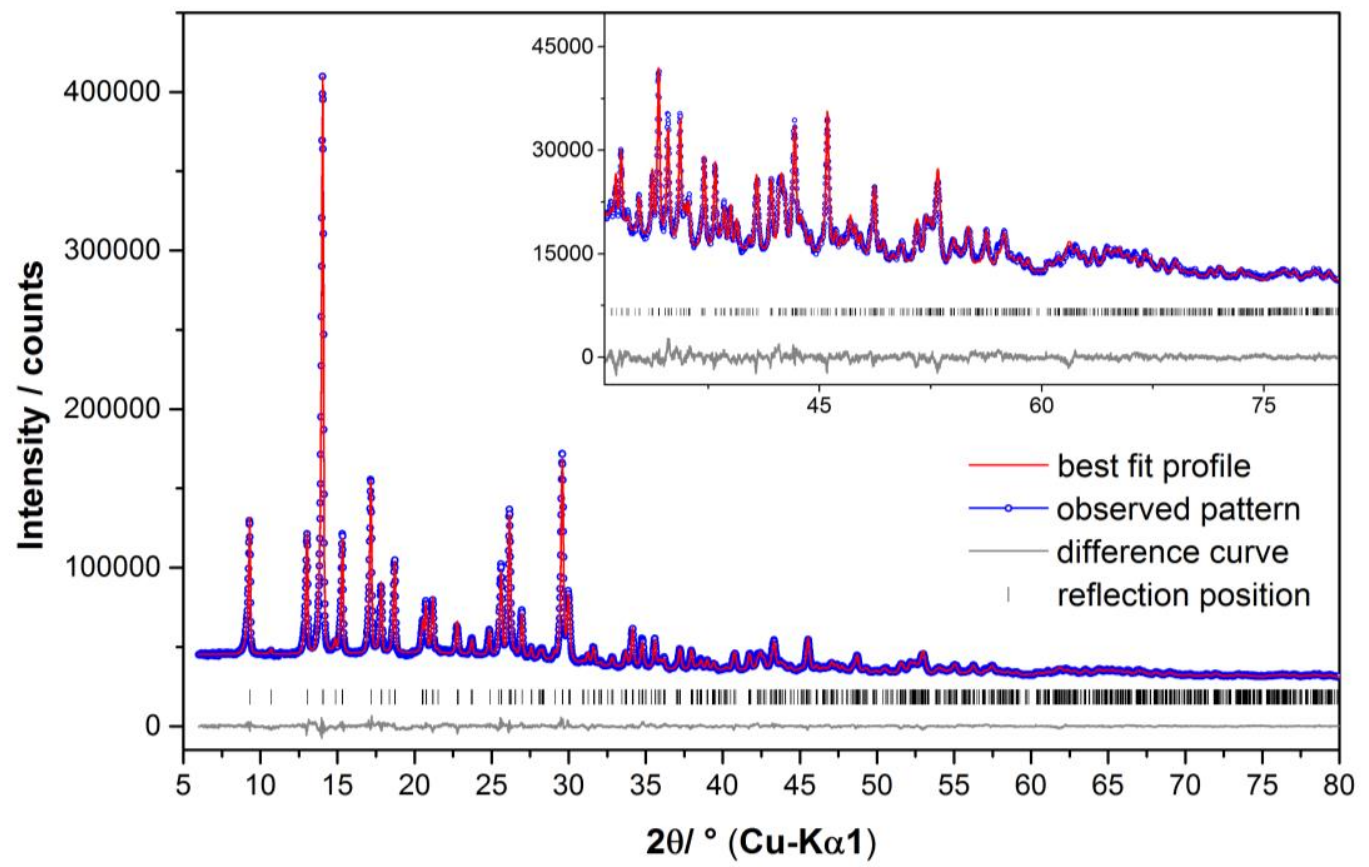

Figure S 17 Scattered X-ray intensities of ZIF-yqt hydrate at $140{ }^{\circ} \mathrm{C}$ measured in a sealed capillary as a function of diffraction angle 20. The observed pattern (circles) measured in Debye-Scherrer geometry, the best Rietveld fit profiles (line) and the difference curve between the observed and the calculated profiles (below) are shown. The high angle part starting at $30.5^{\circ}$ in $2 \theta$ is enlarged for clarity. 


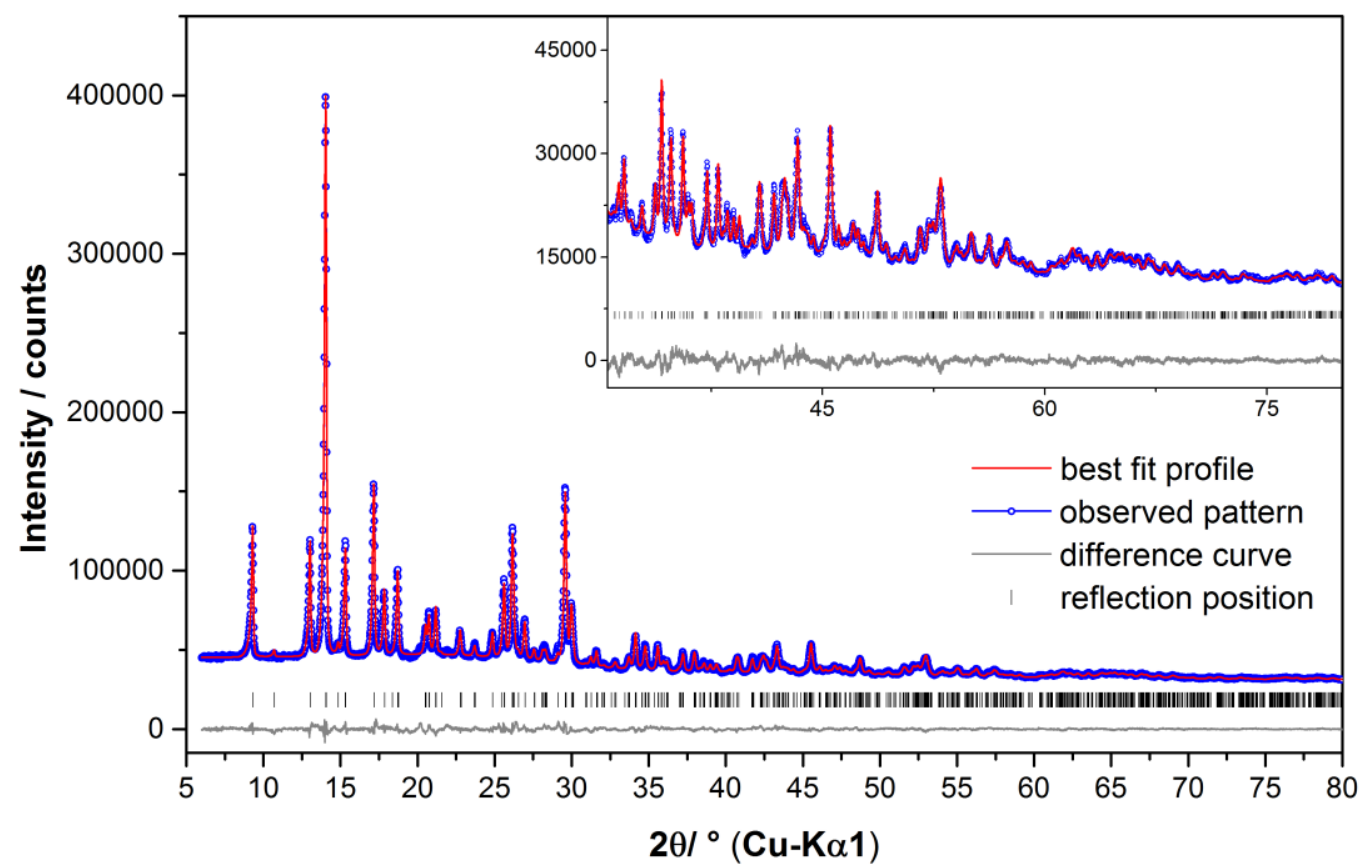

Figure S 18 Scattered X-ray intensities of ZIF-yqt hydrate at $150{ }^{\circ} \mathrm{C}$ measured in a sealed capillary as a function of diffraction angle 2 $\theta$. The observed pattern (circles) measured in Debye-Scherrer geometry, the best Rietveld fit profiles (line) and the difference curve between the observed and the calculated profiles (below) are shown. The high angle part starting at $30.5^{\circ}$ in $2 \theta$ is enlarged for clarity.

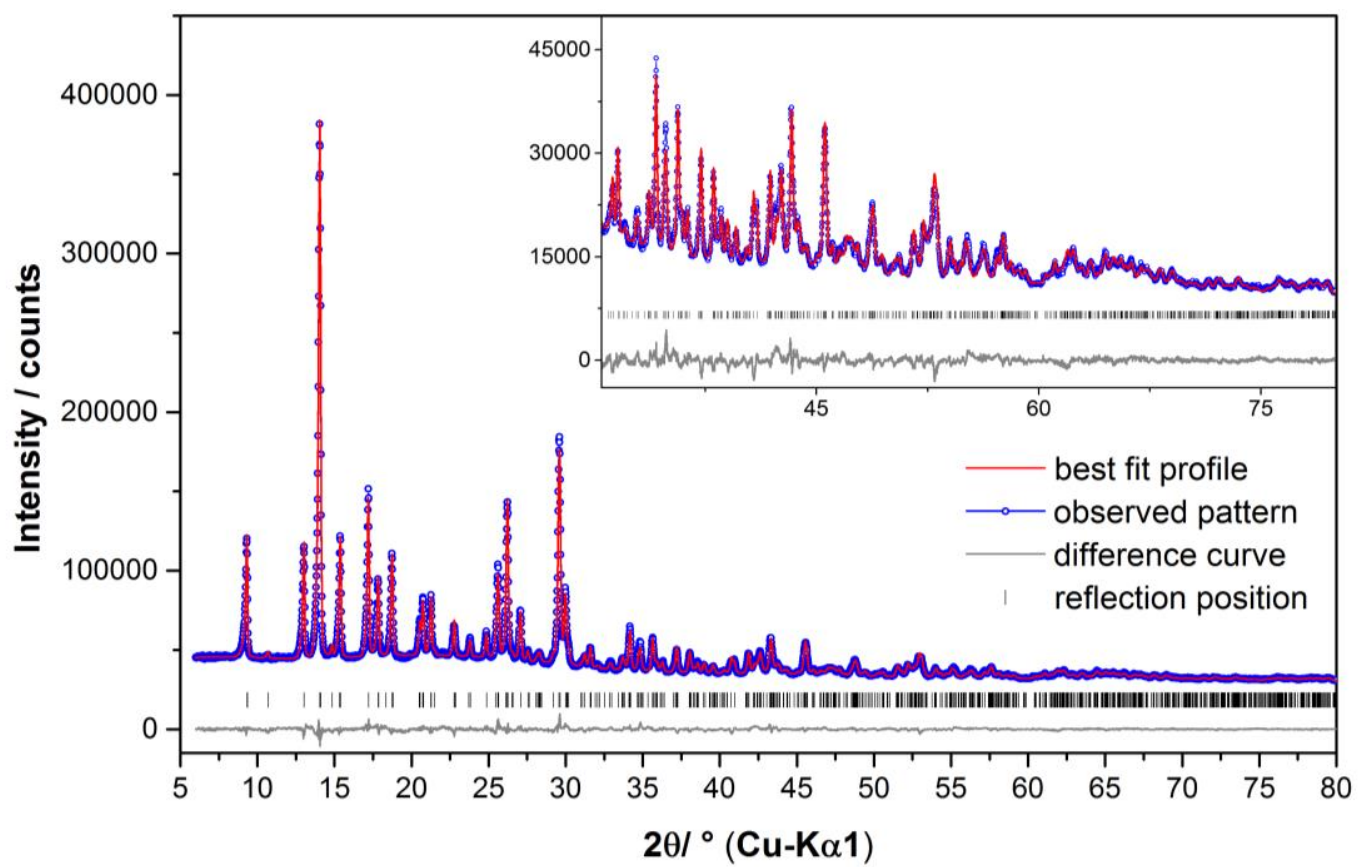

Figure S 19 Scattered X-ray intensities of ZIF-yqt hydrate at $30^{\circ} \mathrm{C}$ measured in an open capillary as a function of diffraction angle $2 \theta$. The observed pattern (circles) measured in Debye-Scherrer geometry, the best Rietveld fit profiles (line) and the difference curve between the observed and the calculated profiles (below) are shown. The high angle part starting at $30.5^{\circ}$ in $2 \theta$ is enlarged for clarity. 


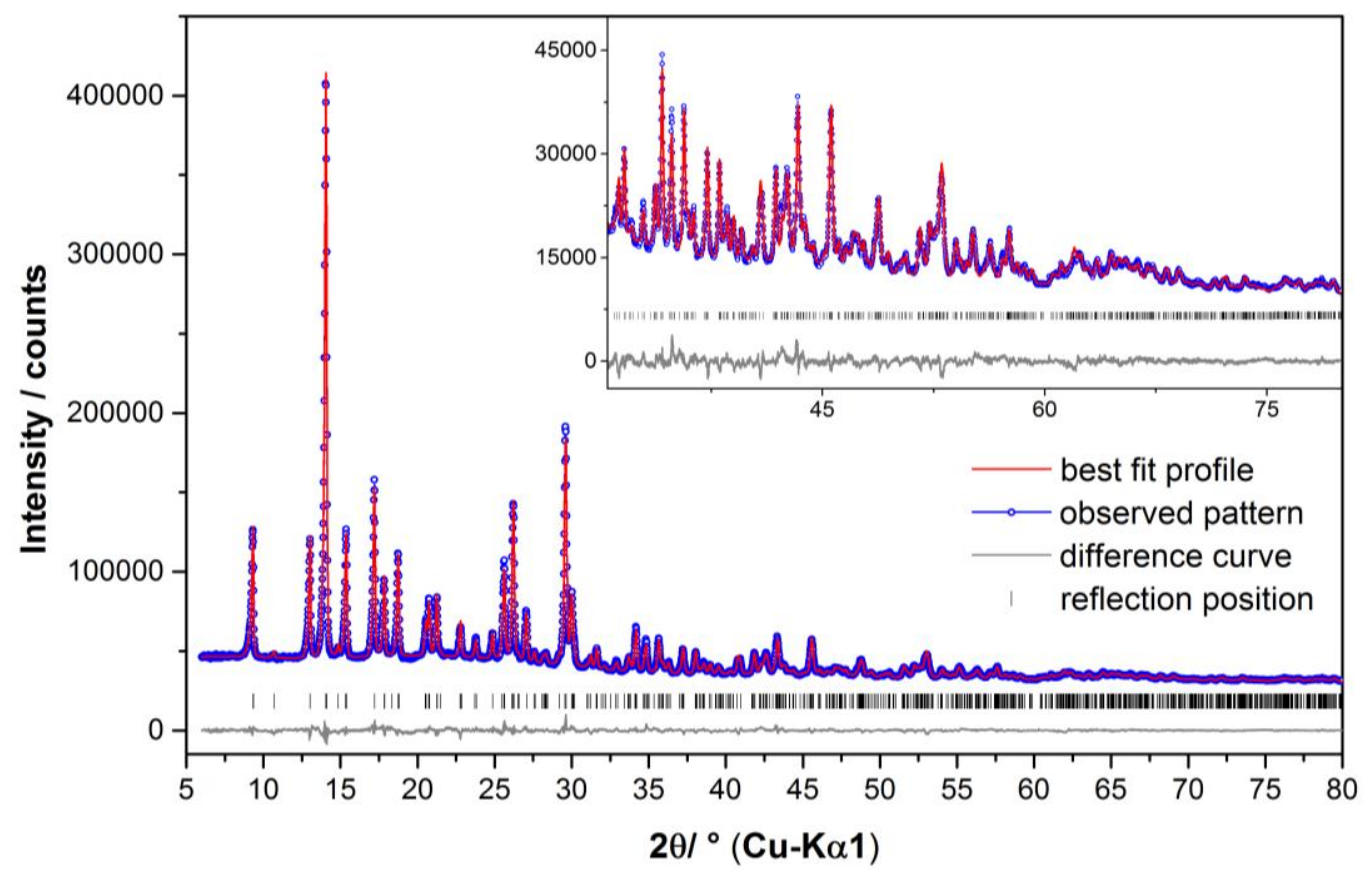

Figure S 20 Scattered X-ray intensities of ZIF-yqt hydrate at $40{ }^{\circ} \mathrm{C}$ measured in an open capillary as a function of diffraction angle $2 \theta$. The observed pattern (circles) measured in Debye-Scherrer geometry, the best Rietveld fit profiles (line) and the difference curve between the observed and the calculated profiles (below) are shown. The high angle part starting at $30.5^{\circ}$ in $2 \theta$ is enlarged for clarity.

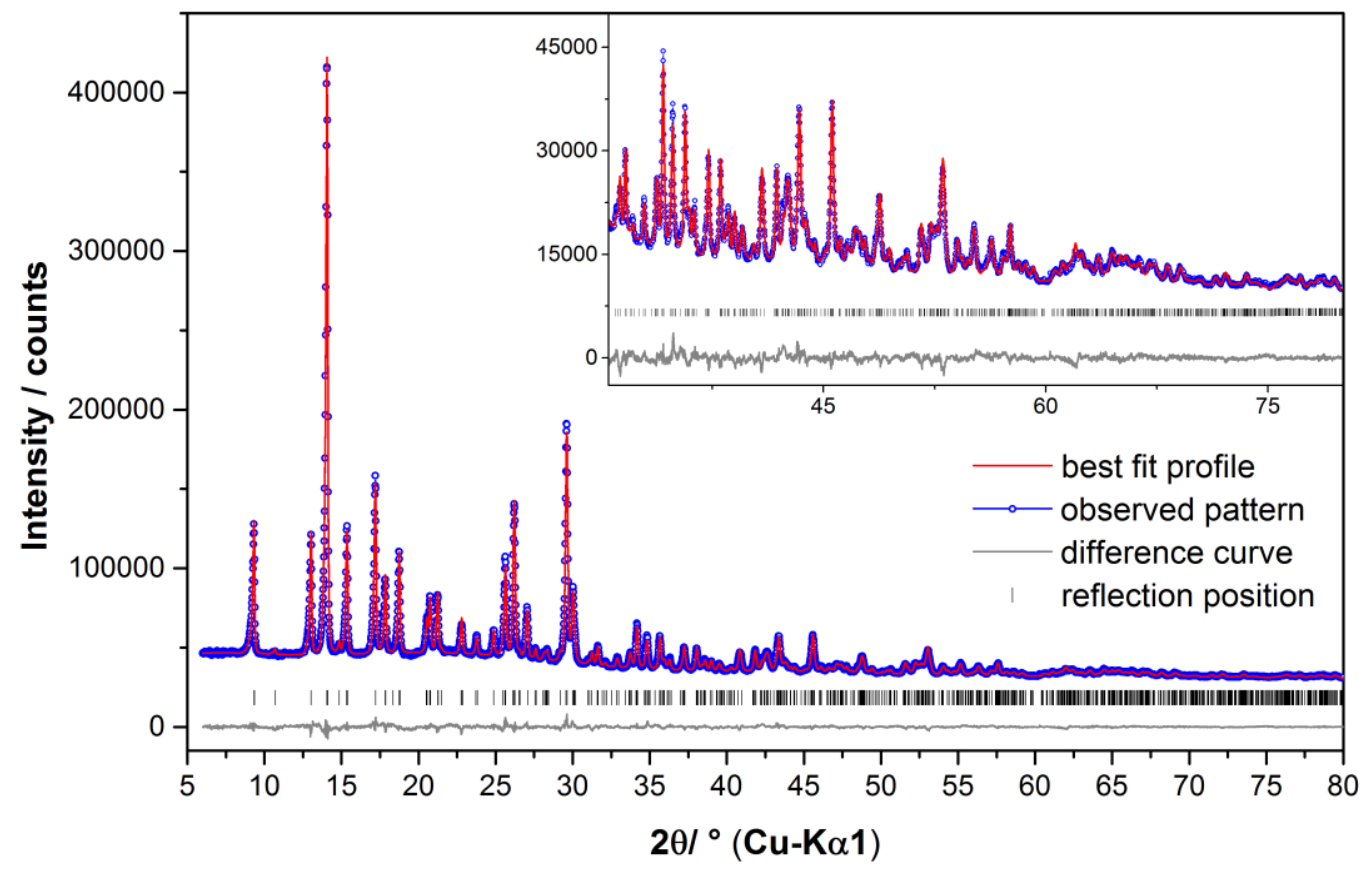

Figure S 21 Scattered X-ray intensities of ZIF-yqt hydrate at $50{ }^{\circ} \mathrm{C}$ measured in an open capillary as a function of diffraction angle 20. The observed pattern (circles) measured in Debye-Scherrer geometry, the best Rietveld fit profiles (line) and the difference curve between the observed and the calculated profiles (below) are shown. The high angle part starting at $30.5^{\circ}$ in $2 \theta$ is enlarged for clarity. 


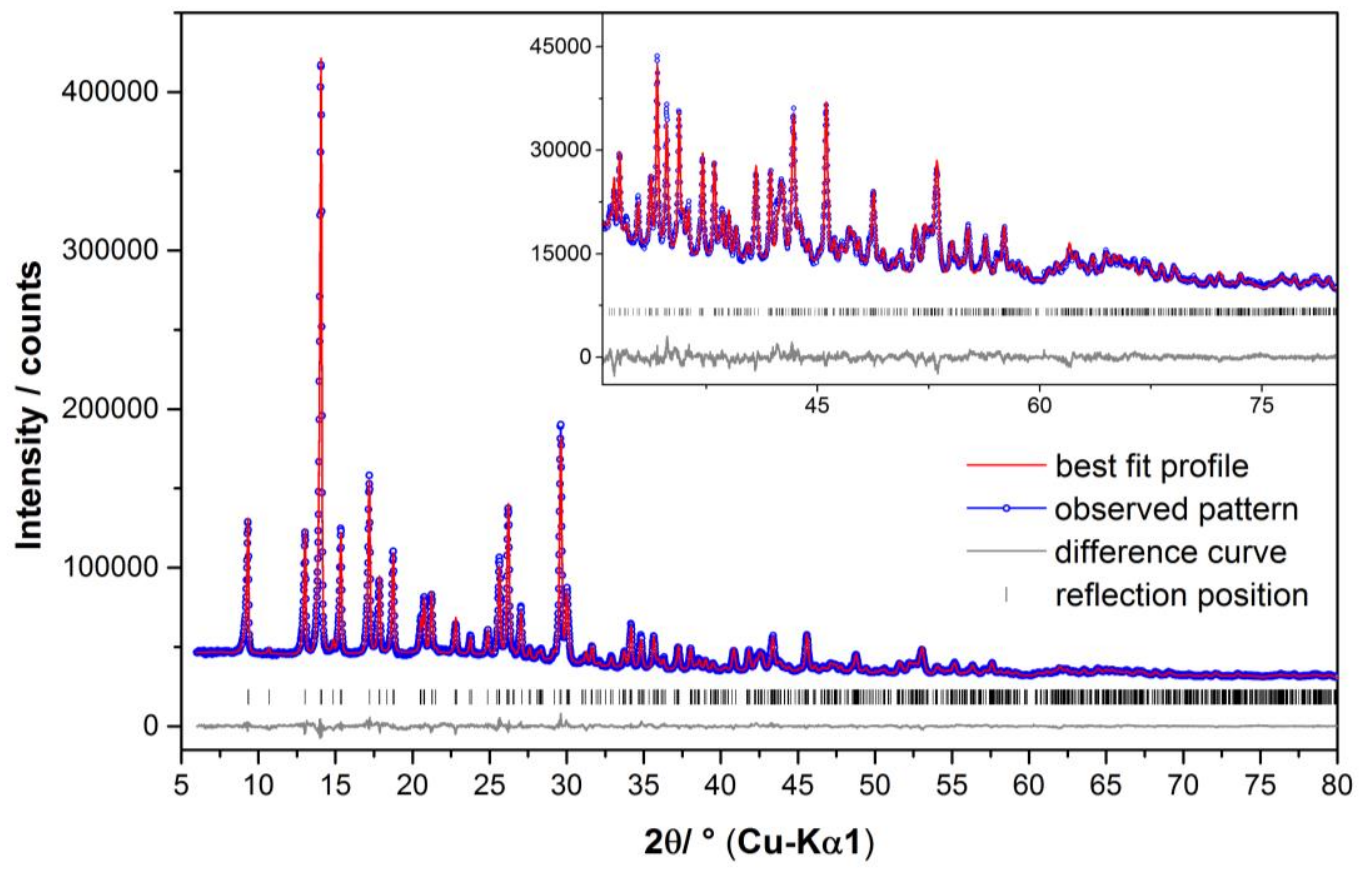

Figure S 22 Scattered X-ray intensities of ZIF-yqt hydrate at $60{ }^{\circ} \mathrm{C}$ measured in an open capillary as a function of diffraction angle $2 \theta$. The observed pattern (circles) measured in Debye-Scherrer geometry, the best Rietveld fit profiles (line) and the difference curve between the observed and the calculated profiles (below) are shown. The high angle part starting at $30.5^{\circ}$ in $2 \theta$ is enlarged for clarity.

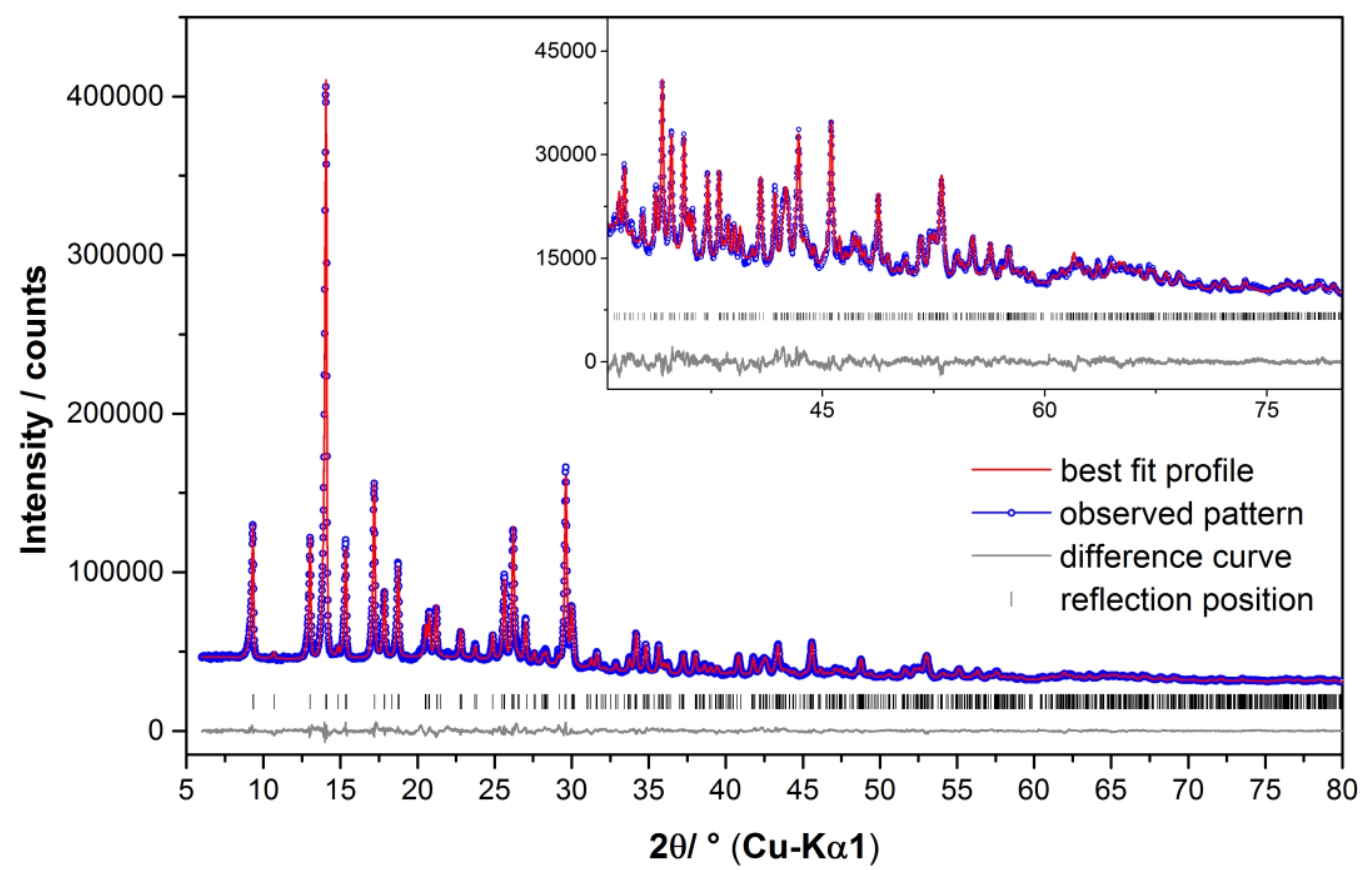

Figure S 23 Scattered X-ray intensities of ZIF-yqt hydrate at $70{ }^{\circ} \mathrm{C}$ measured in an open capillary as a function of diffraction angle $2 \theta$. The observed pattern (circles) measured in Debye-Scherrer geometry, the best Rietveld fit profiles (line) and the difference curve between the observed and the calculated profiles (below) are shown. The high angle part starting at $30.5^{\circ}$ in $2 \theta$ is enlarged for clarity. 


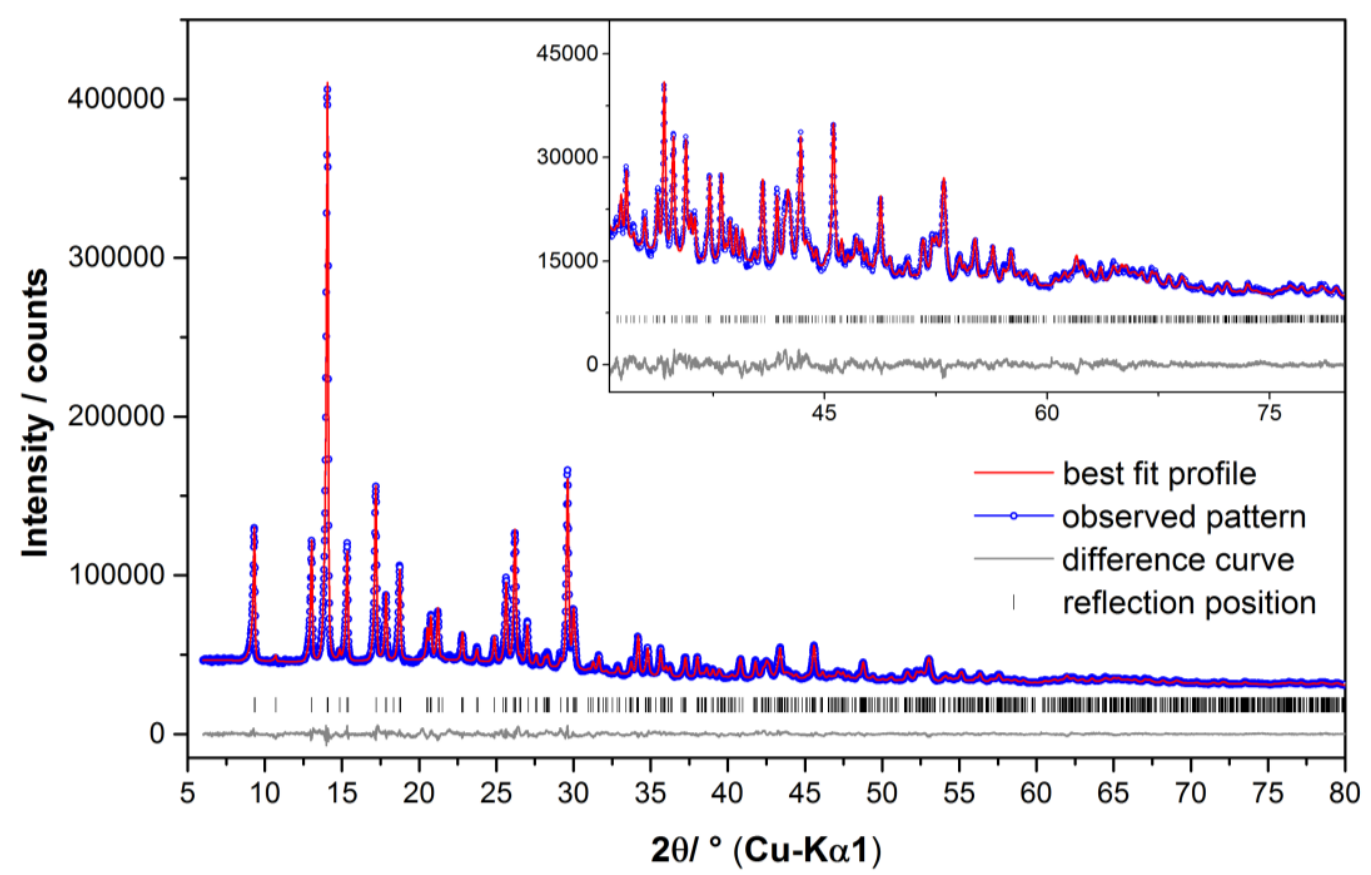

Figure S 24 Scattered X-ray intensities of ZIF-yqt hydrate at $80{ }^{\circ} \mathrm{C}$ measured in an open capillary as a function of diffraction angle 20. The observed pattern (circles) measured in Debye-Scherrer geometry, the best Rietveld fit profiles (line) and the difference curve between the observed and the calculated profiles (below) are shown. The high angle part starting at $30.5^{\circ}$ in $2 \theta$ is enlarged for clarity.

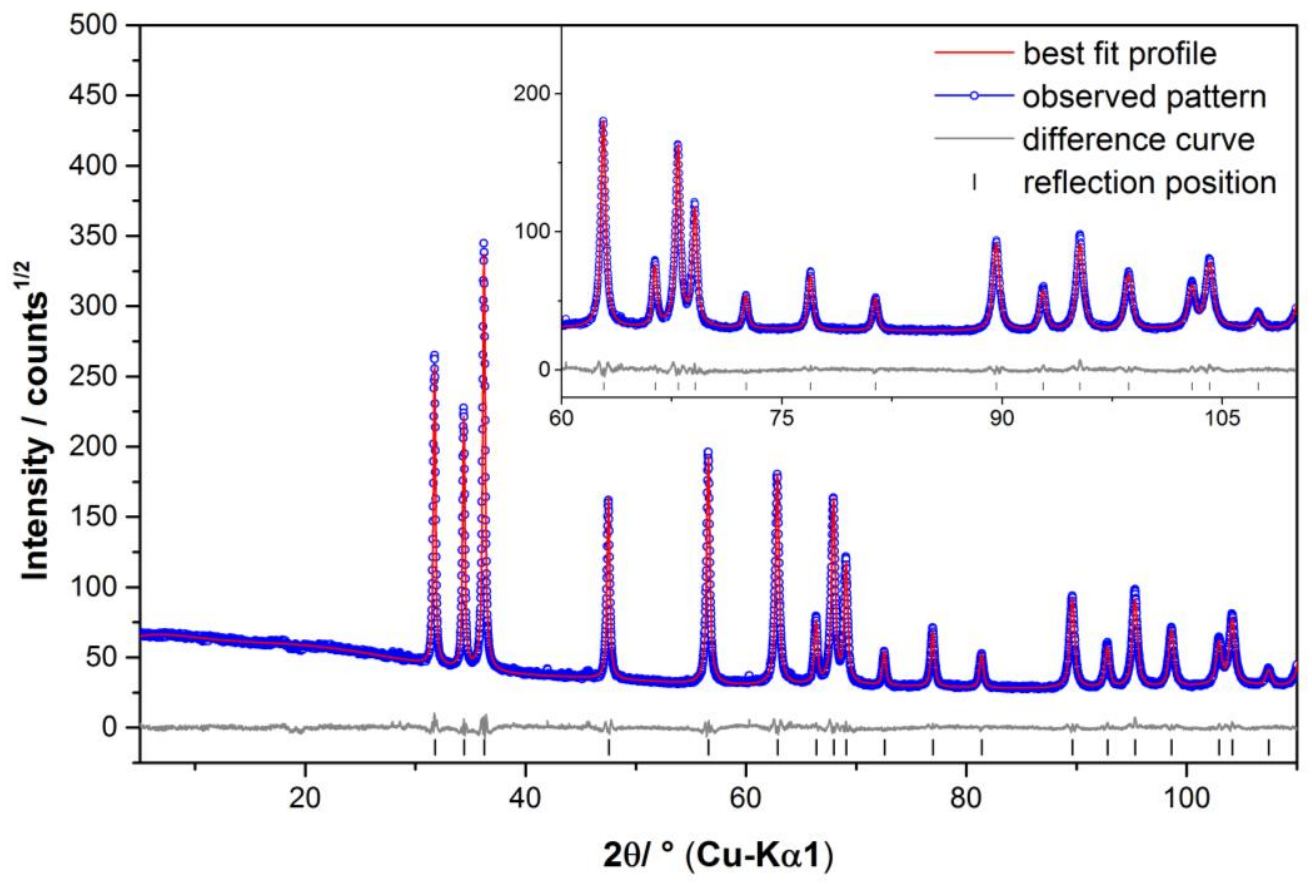

Figure $\mathbf{S} 25$ Scattered X-ray intensities of the residue of ZIF-yqt hydrate after thermal decomposition by heating up to $600{ }^{\circ} \mathrm{C}$ in a nitrogen gas stream measured at ambient conditions as a function of diffraction angle $2 \theta$. The observed pattern (circles) measured in Debye-Scherrer geometry, the best Rietveld fit profiles (line) and the difference curve between the observed and the calculated profiles (below) are shown. The high angle part starting at $60^{\circ}$ in $2 \theta$ is enlarged for clarity. 


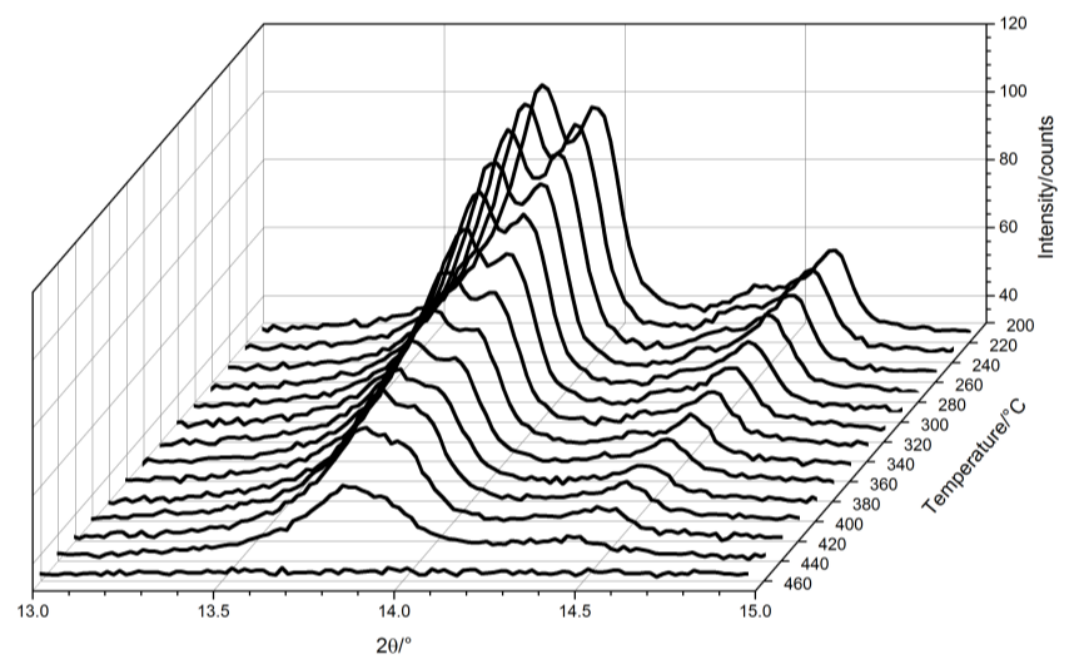

Figure S 26 Excerpts of temperature dependent in-situ XRPD pattern of ZIF-yqt hydrate in the range from $200{ }^{\circ} \mathrm{C}$ to $460{ }^{\circ} \mathrm{C}$ showing the most prominent region of the diffraction pattern of the anhydrous intermediate ZIF-yqt anhydrous.
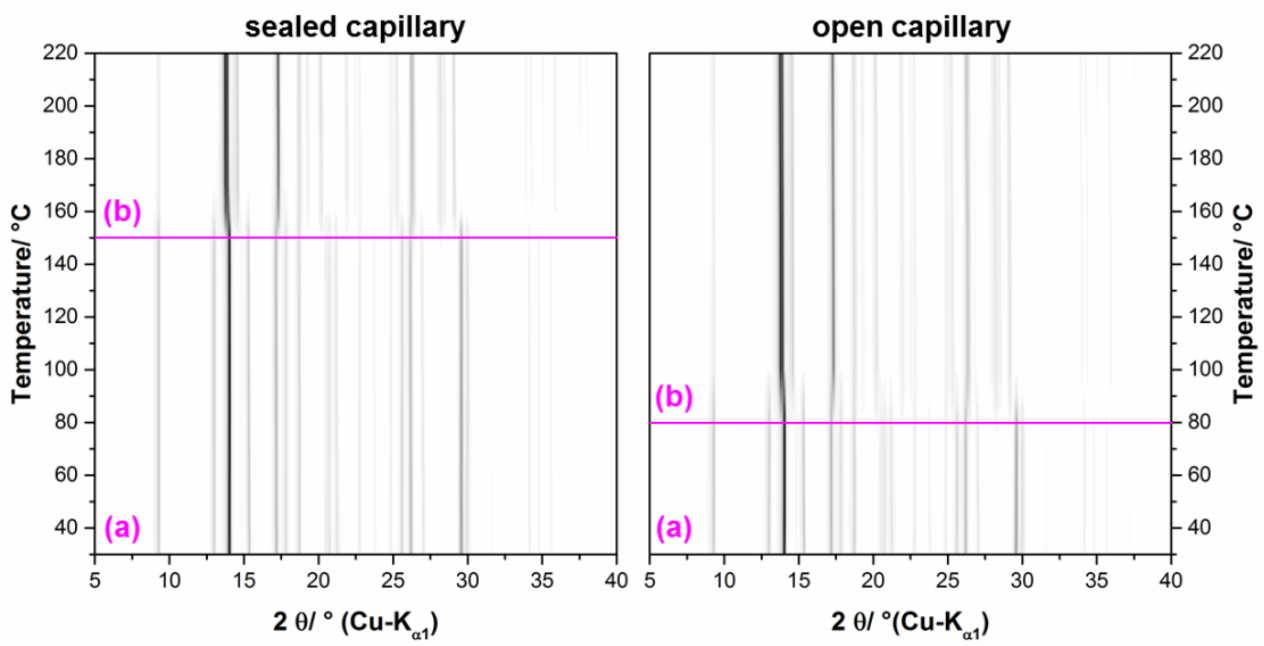

Figure S 27 Comparison of temperature dependent in-situ XRPD measurements of ZIF-yqt hydrate carried out in a sealed and in an open capillary including the assignment of measured reflection positions: (a) ZIF-yqt hydrate (b) ZIF-yqt anhydrous.

\section{Additional Literature:}

(1) Coelho, A. A. TOPAS 6.0; Bruker AXS: Karlsruhe, 2017.

(2) Coelho, A. A. Indexing of Powder Diffraction Patterns by Iterative Use of Singular Value Decomposition. J. Appl. Crystallogr. 2003, 36 (1), 86-95. https://doi.org/10.1107/S0021889802019878.

(3) Pawley, G. S. Unit-Cell Refinement from Powder Diffraction Scans. J. Appl. Crystallogr. 1981, 14 (6), 357-361. https://doi.org/10.1107/S0021889881009618.

(4) Cheary, R. W.; Coelho, A. A.; Cline, J. P. Fundamental Parameters Line Profile Fitting in Laboratory Diffractometers. J. Res. Natl. Inst. Stand. Technol. 2004, 109 (1), 1. https://doi.org/10.6028/jres.109.002. 
(5) Coelho, A. A. Whole-Profile Structure Solution from Powder Diffraction Data Using Simulated Annealing. J. Appl. Crystallogr. 2000, 33 (3), 899-908. https://doi.org/10.1107/S002188980000248X.

(6) Park, K. S.; Ni, Z.; Côté, A. P.; Choi, J. Y.; Huang, R.; Uribe-Romo, F. J.; Chae, H. K.; O’Keeffe, M.; Yaghi, O. M. Exceptional Chemical and Thermal Stability of Zeolitic Imidazolate Frameworks. Proc. Natl. Acad. Sci. 2006, 103 (27), 10186-10191. https://doi.org/10.1073/pnas.0602439103.

(7) Tian, Y.-Q.; Yao, S.-Y.; Gu, D.; Cui, K.-H.; Guo, D.-W.; Zhang, G.; Chen, Z.-X.; Zhao, D.-Y. Cadmium Imidazolate Frameworks with Polymorphism, High Thermal Stability, and a Large Surface Area. Chem. - Eur. J. 2010, 16 (4), 1137-1141. https://doi.org/10.1002/chem.200902729.

(8) Favre-Nicolin, V.; Černý, R. FOX: Modular Approach to Crystal Structure Determination from Powder Diffraction. Mater. Sci. Forum 2004, 443-444, 35-38. https://doi.org/10.4028/www.scientific.net/MSF.443-444.35.

(9) Rietveld, H. M. A Profile Refinement Method for Nuclear and Magnetic Structures. J. Appl. Crystallogr. 1969, 2 (2), 65-71. https://doi.org/10.1107/S0021889869006558.

(10) Chen, R.; Yao, J.; Gu, Q.; Smeets, S.; Baerlocher, C.; Gu, H.; Zhu, D.; Morris, W.; Yaghi, O. M.; Wang, H. A Two-Dimensional Zeolitic Imidazolate Framework with a Cushion-Shaped Cavity for CO2 Adsorption. Chem. Commun. 2013, 49 (82), 9500-9502.

(11) Shi, Q.; Chen, Z.; Song, Z.; Li, J.; Dong, J. Synthesis of ZIF-8 and ZIF-67 by Steam-Assisted Conversion and an Investigation of Their Tribological Behaviors. Angew. Chem. - Int. Ed. 2011, 50 (3), 672-675. 